ARTICLE

https://doi.org/10.1038/s41467-019-09604-0

\title{
Sec14I3 potentiates VEGFR2 signaling to regulate zebrafish vasculogenesis
}

Bo Gong ${ }^{1}$, Zhihao Li ${ }^{1}$, Wanghua Xiao ${ }^{1}$, Guangyuan $\mathrm{Li}^{1}$, Shihui $\mathrm{Ding}^{1}$, Anming Meng ${ }^{1} \&$ Shunji Jia

Vascular endothelial growth factor (VEGF) regulates vasculogenesis by using its tyrosine kinase receptors. However, little is known about whether Sec14-like phosphatidylinositol transfer proteins (PTP) are involved in this process. Here, we show that zebrafish sec14/3, one of the family members, specifically participates in artery and vein formation via regulating angioblasts and subsequent venous progenitors' migration during vasculogenesis. Vascular defects caused by sec14/3 depletion are partially rescued by restoration of VEGFR2 signaling at the receptor or downstream effector level. Biochemical analyses show that Sec14I3/SEC14L2 physically bind to VEGFR2 and prevent it from dephosphorylation specifically at the $\mathrm{Y}^{1175}$ site by peri-membrane tyrosine phosphatase PTP1B, therefore potentiating VEGFR2 signaling activation. Meanwhile, Sec14I3 and SEC14L2 interact with RAB5A/4A and facilitate the formation of their GTP-bound states, which might be critical for VEGFR2 endocytic trafficking. Thus, we conclude that Sec1413 controls vasculogenesis in zebrafish via the regulation of VEGFR2 activation.

\footnotetext{
${ }^{1}$ State Key Laboratory of Membrane Biology, Tsinghua-Peking Center for Life Sciences, School of Life Sciences, Tsinghua University, 100084 Beijing, China. Correspondence and requests for materials should be addressed to A.M. (email: mengam@mail.tsinghua.edu.cn) or to S.J. (email: jiasj@mail.tsinghua.edu.cn)
} 
T he vertebrate vasculature, as a tree-like tubular and highly dynamic plexus, extends into almost all tissues for a constant supply of nutrients and oxygens, or transport of metabolic wastes ${ }^{1,2}$. The formation of a functional vascular system is essential for embryonic development, and its structural abnormalities always lead to pathological diseases ${ }^{3,4}$. This systematic and hierarchical vascular system is achieved by two distinct mechanisms, vasculogenesis (de novo assembly of vessels) and angiogenesis (modification and expansion of pre-existing vessels). In zebrafish, angioblasts derived from the lateral plate mesoderm eventually give rise to the first embryonic artery (dorsal aorta, DA) and vein (posterior cardinal vein, PCV) during vasculogenesis, and then these vascular systems are rapidly expanded and remodeled during angiogenesis to consummate vessel networks, including the formation of intersegmental veins (ISV) by endothelial cell sprouting ${ }^{2,5-7}$.

So far, a variety of signaling molecules and transcription factors have been implicated in the formation of the vertebrate vasculature via regulating endothelial cell proliferation, differentiation, migration, and position ${ }^{2,4,8}$. Vascular endothelial growth factor (VEGF) signaling is considered as the most critical and pivotal one during embryonic vasculogenesis as well as angiogenesis ${ }^{9,10}$. After secretion, VEGF ligands bind in an overlapping pattern to three receptor tyrosine kinases (RTKs), known as VEGFR1/ Flt-1, VEGFR2/Flk-1/KDR, and VEGFR3/Flt-4 on the plasma membrane, followed by receptor dimerization and autophosphorylation at particular tyrosine sites. Then, the phosphorylated receptors recruit interacting proteins and further trigger the activation of downstream cascades via PLC $\gamma /$ ERK and PI3K/AKT pathways ${ }^{11}$. Among these VEGFRs, VEGFR2 is considered as the major mediator of proangiogenic signaling in almost all aspects of vascular-endothelial-cell biology ${ }^{8}$. Of particular interest, in endothelial cells, VEGFR2 displays distinct distributions in subcellular pools, including cell surface, endocytic storage compartments, lipid rafts as well as cell-cell junctions ${ }^{12-14}$. As a result, VEGFR2 signaling could be monitored on the plasma membrane or within endosomes. However, what determines the activation of a specific pool is poorly understood ${ }^{8}$.

To achieve specific signal outputs with coordinated duration and amplitude, VEGFR2 signaling is strictly regulated at numerous levels, such as the receptor expression level, the availability, and affinities for binding its different ligands, the presence of co-receptors and repressor (tyrosine phosphatases), and so on ${ }^{15-17}$. More importantly, the intracellular trafficking and endocytic kinetics of VEGFR2 also regulate the signal outputs significantly ${ }^{18-20}$. Upon ligand binding, VEGFR2 is internalized mainly in a clathrin-dependent manner with the help of motor proteins and trafficked to RAB5-positive and EEA1-positive early endosomes. Unless VEGFR2 is dephosphorylated by the perimembrane resident PTP1B at the $\mathrm{Y}^{1175}$ site $^{21}$, these VEGFR2containing vesicles can either be targeted for degradation via the RAB7-pathway to attenuate the signaling or recycled back to the plasma membrane via the fast (RAB4-dependent) or slow (RAB11-dependent) route for further potentiation ${ }^{19}$. Although NRP1-synectin-myoVI and ephrinB2-DAB2-PAR3 complex have been demonstrated to promote endosome movements into cell $^{22,23}$, many events involved in VEGFR2 internalization and trafficking are still unclear. Full understanding of the endocytic VEGFR2 trafficking will further advance our understanding of the VEGF signaling and the consequential biological functions.

Sec1413 proteins belong to phosphatidylinositol transfer proteins (PITPs), which were first described as transporters to potentiate phosphatidylinositol (PI) and phosphatidylcholine (PC) exchange between membranes in vitro ${ }^{24}$. Because of the involvement of PI molecules in endocytic membrane trafficking, PITPs are also proposed to play a vital role in vesicle budding from the trans Golgi network (TGN) as well as in priming of exocytosis ${ }^{25-27}$. Although it is reported that there is a crosstalk between PITPs with Wnt/ $\mathrm{Ca}^{2+}$ and EGF signaling ${ }^{28-30}$, whether PITPs integrate lipid metabolism with intracellular VEGF signaling has not been studied.

In this paper, we demonstrate that Sec14l3 regulates VEGF signaling at its receptor level and participates in vascular development of zebrafish embryos. Using morpholino knockdown in combination with CRISPR/Cas9 knockout system, we find that sec14l3 deficiency results in narrowed DA and PCV lumens. Mechanistically, Sec14l3 promotes RAB5A/4A activation to accelerate VEGFR2 internalization and recycling, thus preventing VEGFR2 from exposure to the phosphatase activity of PTP1B, which ultimately enhances the downstream VEGF signaling. It will be meaningful to interpret PITPs function in regulating VEGFR2 trafficking as well as in understanding VEGF signaling during vertebrate vasculature development.

\section{Results}

Sec1413/SEC14L2 are enriched in the endothelial cells. Vascular system formation is important for the normal development of vertebrate embryos, as well as for the survival of adults ${ }^{4}$. In order to identify critical genes required for zebrafish vasculature development, we isolated GFP-positive cell populations, by fluorescence-activated cell sorting (FACS), from $\operatorname{Tg}(f l i 1 a: E G F P)^{y 1}$ embryos $^{7}$ at $24 \mathrm{hpf}$, when the DA has separated from the PCV 5,6 RNA sequencing of the isolated cells showed that genes in vascular, hematopoietic and pharyngeal arch cells were highly expressed as reported previously (Supplementary data 1$)^{7}$. Of our interest is the PITP gene sec14l3. Whole-mount in situ hybridization (WISH) results showed that $\sec 14 l 3$ was spatially expressed in vasculature at $24 \mathrm{hpf}$ (Fig. 1a). Transverse sectioning post WISH indicated that sec14l3 is expressed in both arterial and venous endothelial progenitor cells at $19 \mathrm{hpf}$, and thereafter it is restricted to the PCV compartment at $25 \mathrm{hpf}$ (Fig. 1a). The expression of sec14l3 in endothelial cells is almost eliminated in etsrp/etv2 mutants lacking trunk vasculature (Fig. 1b) ${ }^{31}$, which confirms its vascular expression. Quantitative RT-PCR analysis indicated that sec14l3 expression level in $\mathrm{GFP}^{+}$cells from $\mathrm{Tg}$ (fli1a:EGFP)y ${ }^{y 1}$ embryos at $24 \mathrm{hpf}$ is enriched by about 5 folds, which is comparable to that of $k d r l / v e g f r 2$, a specific vascular marker gene (Fig. 1c).

To know whether the enrichment of sec14l3 in endothelial cells is conserved in mammals, we analyzed human SEC14L2/SEC14L3 expression in human umbilical vein endothelial cells (HUVECs). RT-PCR results showed that SEC14L2 transcripts exist while SEC14L3 does not (Supplementary Fig. 1a). Importantly, immunostaining data demonstrated that SEC14L2 protein locates throughout the cells, and presents as vesicles in the cytosol. Given the fact that intracellular VEGFR2 mainly distributes to endosomes and Golgi ${ }^{12,32}$, we co-stained our protein with VEGFR2 and it showed that about 28\% of SEC14L2 is co-localized with VEGFR2 in the endocytic pool in HUVECs (Fig. 1d). Taken together, these data suggest a possible implication of zebrafish sec14l3/human SEC14L2 in vascular development.

Sec1413/SEC14L2 are required for vascular formation. To investigate whether Sec1413 is required for vasculature development during zebrafish embryogenesis, two antisense morpholino oligonucleotides (MO), sec1413-tMO2 and sec14l3-sMO, were designed to knock down sec14l3 expression in vivo. sec1413-tMO2 targets the $5^{\prime}$ untranslated region of sec14l3 mRNA to block its translation, while sec14l3-sMO targets the 2nd exon-intron junction to interrupt normal splicing of the primary transcript. Their effectiveness was confirmed by previous report ${ }^{30}$ and 


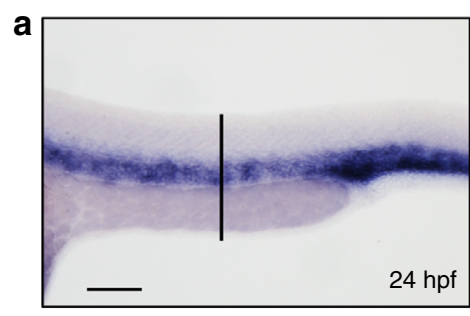

b
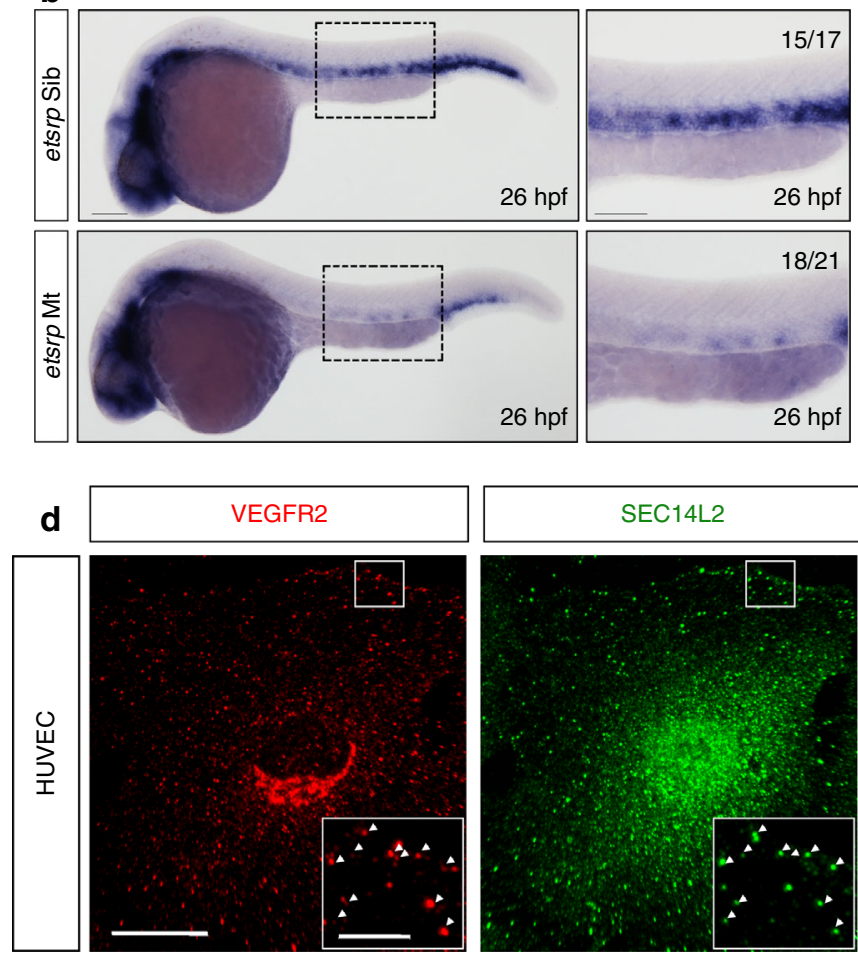
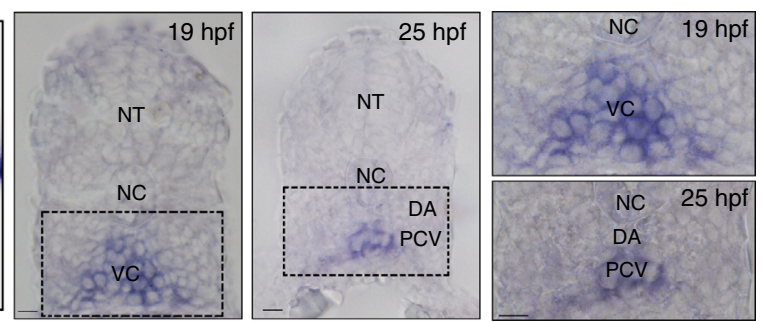

C
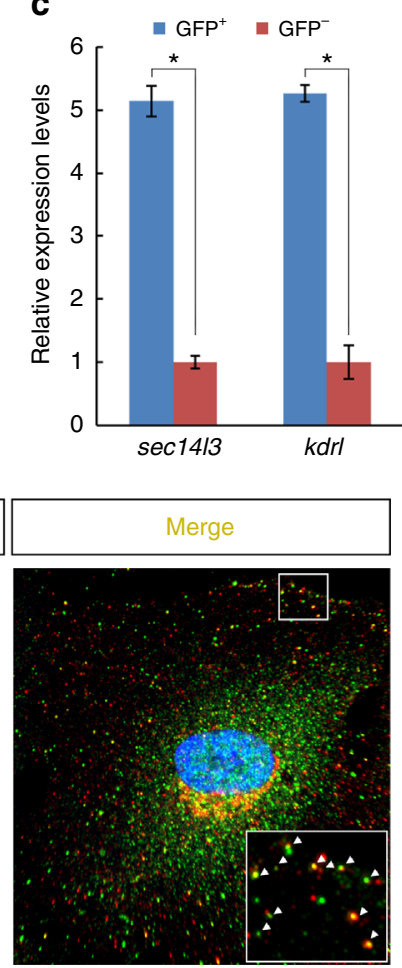

Fig. 1 sec14I3/SEC14L2 are expressed in endothelial cells and co-localized with VEGFR2. a Expression pattern of sec14/3 examined by WISH. Trunk vascular expression of sec14/3 is shown at $24 \mathrm{hpf}$ and the vertical line denotes the equivalent position of transverse sections in embryos at $19 \mathrm{hpf}$ and $25 \mathrm{hpf}$. Highresolution views of the boxed regions are shown at right panels. Adjacent tissues are denoted. NT, neural tube; NC, notochord; DA, dorsal aorta; PCV, posterior cardinal vein; VC, vascular cord. Scale bars, $100 \mu \mathrm{m}$. b The absence of sec14/3 expression in the trunk vascular system of etsrp mutant embryos at $26 \mathrm{hpf}$. High-resolution views of the boxed regions are shown at right panels. Scale bars, $100 \mu \mathrm{m}$. c Quantitative RT-PCR of sec14/3 mRNA in both GFP + cells and GFP- cells from $T g$ (flita:EGFP)y ${ }^{1}$ transgenic embryos at 24 hpf. sec14/3 is highly enriched in GFP+ cells (blue bars) relative to negative cells (red bars), using $\beta$-actin as the internal control. kdrl/vegfr 2 mRNA serves as a positive control for vascular endothelial cells. A two-tailed $t$-test was used for statistical analysis. ${ }^{*} p<0.05$, the exact $p$-values in each figure are shown in the Source data file. d SEC14L2 is co-localized with VEGFR2 in HUVECs. Red and green represent anti-VEGFR2 antibody and anti-SEC14L2 antibody staining signal respectively; blue symbolizes DAPI stained nucleus. Regions in the boxes are enlarged in the right corner. Scale bars, $20 \mu \mathrm{m}$ in the original pictures and $5 \mu \mathrm{m}$ in the enlarged panels

RT-PCR analysis (Supplementary Fig. 1b). Compared to control embryos injected with standard MO (std-MO), 5 ng sec14l3tMO2 injection showed no obvious difference in morphology, with normal convergent and extension movements at gastrulation stages (Supplementary Fig. 1c). When analyzing vascular development, we found that knockdown of sec14l3 in $\mathrm{Tg}(\mathrm{kdrl}$ : $G F P)^{s 843 T g}$ embryos resulted in arterial-venous segregation and luminal expansion defects with compromised ISVs formation (Fig. 2a and Supplementary Fig. 2a). However, when sec14l3tMO2 was injected in a p53 mutant background, ISVs sprouting defect was largely restored at $48 \mathrm{hpf}$ (Fig. 2a and Supplementary Fig. 2b), which presumably relieves the general off-target effect of morpholinos ${ }^{33}$. Therefore, we conclude that sec $14 l 3$ specifically regulates DA and PCV formation during vasculogenesis.

To figure out sec14l3 functions in a maternal- or zygoticdependent manner, we turned to use the splicing blocker sec1413sMO. Consistent with sec14l3-tMO2, sec1413-sMO injection similarly inhibited the arterial-venous segregation at $23 \mathrm{hpf}$ and luminal expansion at $25 \mathrm{hpf}$ (Fig. 2b, c), suggesting that the vascular defects mainly arise from the loss of zygotic sec14l3. More importantly, the DA and PCV defects in sec14l3 morphants were restored by sec14l3 mRNA overexpression (Fig. 2b, c), indicative of specific effects.

To substantiate the knockdown effect, we also generated a sec14l3 mutant based on clustered regularly interspaced short palindromic repeats (CRISPR)/Cas9 technology, using a guide RNA targeting the 5th exon of sec14l3 gene in $\mathrm{Tg}(\mathrm{kdrl}: \mathrm{GFP})^{\mathrm{s} 843 \mathrm{Tg}}$ transgenic line (Supplementary Fig. 3a). The isolated mutant allele contains a 10-bp deletion near the target site, causing a premature stop codon and presumably a truncated protein only owning CRAL-TRIO domain (Supplementary Fig. 3a). Although there is no obvious difference between mutant and wild-type embryos in morphology (Supplementary Fig. 3b), sec14l3 transcripts are almost abolished in homozygous mutants 

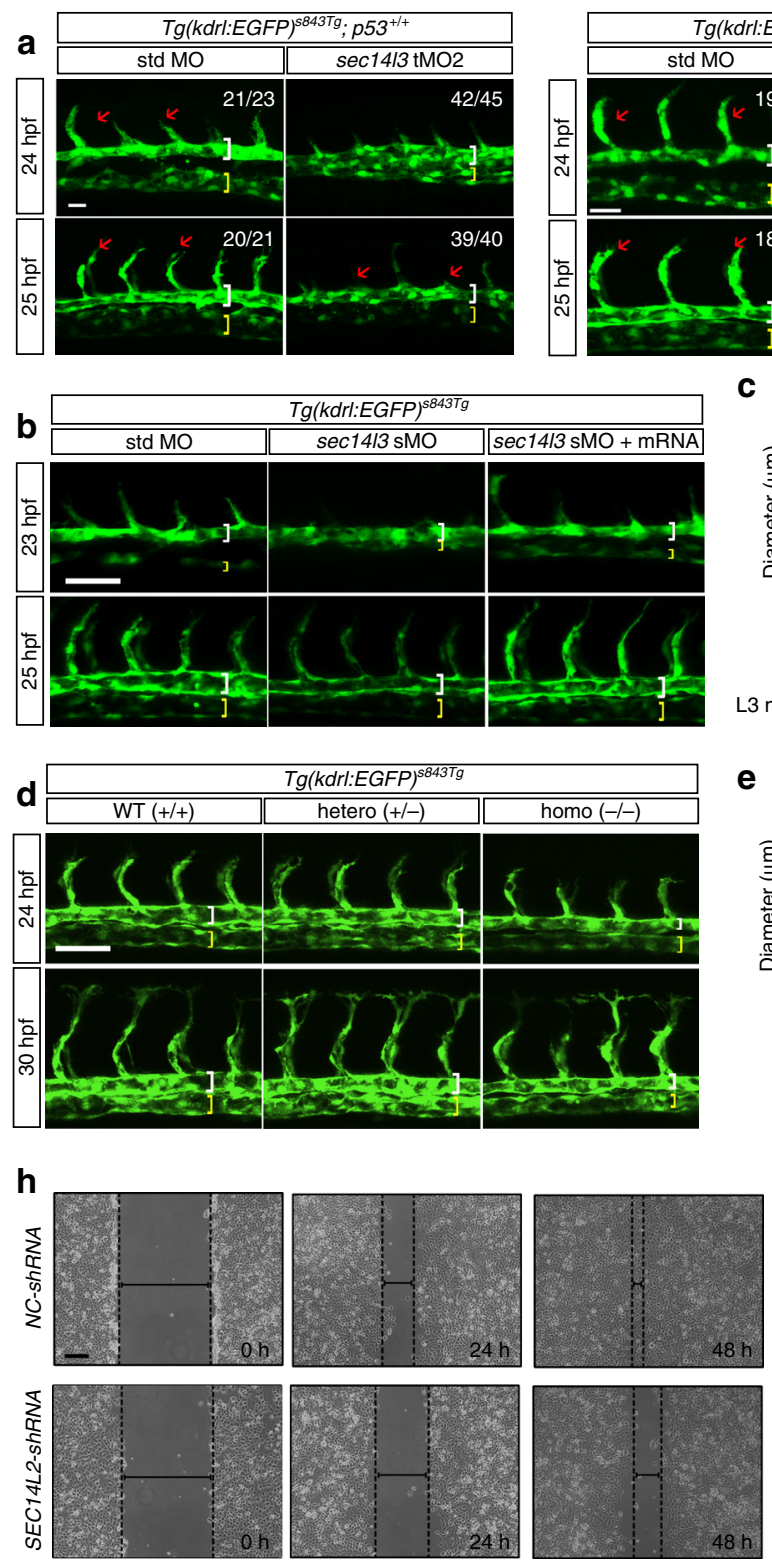
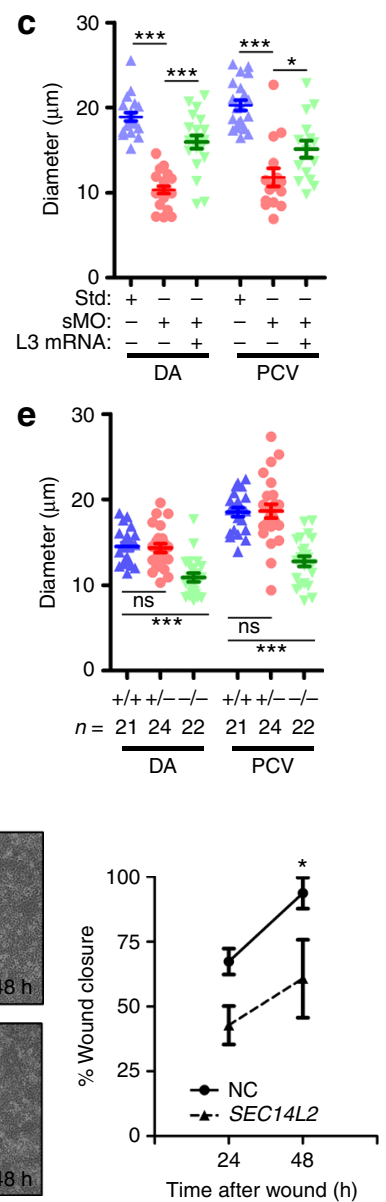
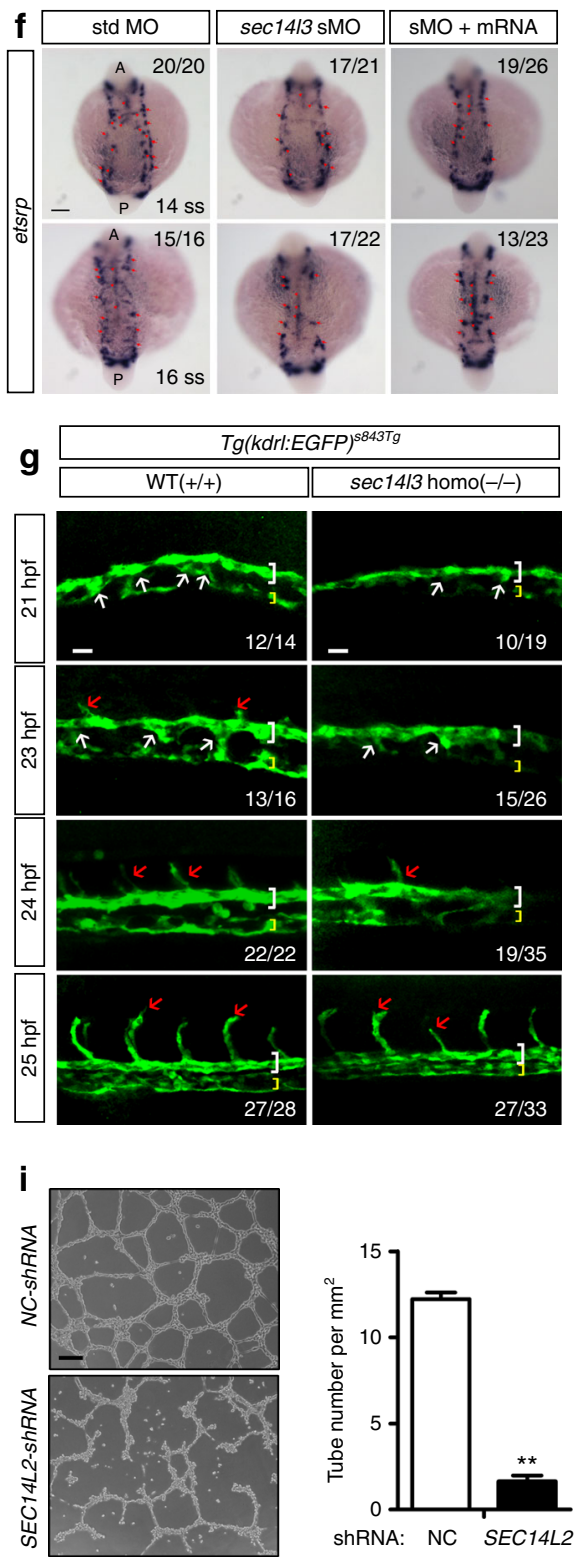

Fig. 2 sec14/3/SEC14L2 are required for vascular formation in vivo and in vitro. a sec14I3-tMO injection impairs arterial-venous segregation and luminal formation in zebrafish. Embryos of $T g(k d r l: E G F P)$ s843Tg; $553^{+/+}$(left panel) or $T g(k d r l: E G F P)$ s843Tg;p53-/- fish (right panel) were used. White and yellow brackets indicate DA and PCV respectively; red arrows indicate ISVs sprouting. The ratio in the right corner indicates the number of embryos with observed pattern/the total number of observed embryos. $\mathbf{b}$ sec14l3-sMO mediated knockdown of sec14/3 causes trunk vascular defects. $0.5 \mathrm{ng}$ sec14/3-sMO and $150 \mathrm{pg}$ sec14/3 mRNA were co-injected for rescue experiment. c Statistical analyses of DA and PCV diameters at $25 \mathrm{hpf}$ in $\mathbf{b}$ ( $n=20$ embryos). d Vasculature defects in sec14/3 mutant embryos. Heterozygous mutants were intercrossed and embryos were harvested for vasculature observation and genotyping analysis individually. e Statistical analyses of DA and PCV diameters at $30 \mathrm{hpf}$ in $\mathbf{d}$. The total number of embryos in each genotype is indicated. f sec14/3-sMO injection causes angioblast migration defects. $0.5 \mathrm{ng}$ sec14/3-sMO and $100 \mathrm{pg}$ sec14/3 mRNA were co-injected for rescue experiment. WISH using etsrp probe marks the medial (arrowhead) and lateral (arrow) progenitor populations simultaneously. $\mathbf{g}$ Ventral sprouting defects in sec14/3 mutant embryos. h SEC14L2 knockdown inhibits the in vitro wound closure process. HUVECs infected with control or SEC14L2 shRNA were used for wound closure observation. The ratio of recovered wound width at a specific time point post-wounding to the initial wounding width was calculated. Dashed lines indicate wound edges. Statistical data are shown $(n=3)$. i SEC14L2 knockdown inhibits in vitro tube formation. HUVECs were infected with shRNA-packed lentivirus and the complete tube number was scored per field at $24 \mathrm{~h}$ after plating. The statistical data are shown on the right $(n=3$ independent experiments, 60 fields total). All statistical data are shown as mean \pm SEM. One-way ANOVA tests were used for statistical analyses in $\mathbf{c}$, e and $t$-tests were used for $\mathbf{h}-\mathbf{i}$. ${ }^{\star} p<0.05$; ${ }^{\star \star} p<0.01 ;{ }^{\star \star \star} p<0.01$; ns, not significant. Scale bars, $25 \mu \mathrm{m}$ for $\mathbf{a}, 50 \mu \mathrm{m}$ for $\mathbf{b}, \mathbf{d}, \mathbf{g}$, and $\mathbf{h}-\mathbf{i}, 100 \mu \mathrm{m}$ for $\mathbf{f}$. Source data are provided as a Source Data file

(Supplementary Fig. 3c). Compared with wild-type and heterozygous, homozygous embryos exhibit arterial-venous segregation and luminal expansion defects with nearly normal ISVs formation, distinct from impaired ISV length in sec14l3 morphants (Fig. 2d, e, and Supplementary Fig. 4). This is partially due to a non-specific effect of tMOs, as after injection of sec1413-tMO2 in homozygotes, it could not cause further DA and PCV defects, but with further reduction of ISV length (Supplementary Fig. 4). Therefore, we conclude that sec14l3 is required for zebrafish DA and PCV formation. 
Since it is reported that two distinct mechanisms could account for the DA and PCV formation in zebrafish ${ }^{34,35}$, we wonder what happens in our mutants. Using fli and etsrp as probes, the WISH result showed that in sec14l3 deficient embryos, the specification of angioblast is not affected at 8-somite stages (Supplementary Fig. 5), while its migration is significantly reduced at 14-16 somite stages, which could be rescued by the injection of sec14l3 mRNA (Fig. 2f). Meanwhile, with highresolution imaging in $T g(k d r l: G F P)^{s 843 T g}$ embryos, we found that the sprouting of venous progenitors from the vascular cord is also decreased from 21 to $23 \mathrm{hpf}$ in sec14l3 deficient embryos (Fig. $2 \mathrm{~g}$ and Supplementary Fig. 6). Therefore, we'd like to conclude that sec14l3 play important roles in zebrafish DA and PCV blood vessels formation via regulating angioblast migration.

Since SEC14L2 is expressed in HUVECs (Fig. 1d and Supplementary Fig.1a), we assessed its function by in vitro wound closure assay in HUVECs. Compared with NC-shRNA infected control, the wound gap in SEC14L2 shRNA cells was closed much slower (Fig. 2h), suggesting a requirement of SEC14L2 for endothelial cell migration. The in vitro tube formation assay in HUVECs ${ }^{36}$ revealed that knockdown of SEC14L2 obviously inhibited the formation of capillary-like tubes (Fig. 2i), indicating a facilitating effect of SEC14L2 on the tube formation of endothelial cells. Therefore, SEC14L2 may mainly promote mammalian endothelial cell migration to maintain vasculature formation.

Sec1413/SEC14L2 promote VEGF-induced VEGFR2 activation. VEGFR2 signaling plays vital roles during zebrafish vasculature, which could be motivated both by VEGF ligands and blood flow-induced fluid shear stress (FSS) ${ }^{10,37}$. Given that SEC14L2 co-localizes with VEGFR2 in HUVECs (Fig. 1d), we hypothesized that SEC14L2 regulates VEGFR2 signaling. Since Sec14l3 exerts its function on angioblast migration during vasculogenesis before the onset of blood flow in vivo (Fig. 2f, g and Supplementary Fig. 6), we focused on the VEGF- but not FSS-induced VEGFR2 signaling transduction in vitro, and further investigated SEC14L2 knockdown effect in HUVECs on the downstream effectors of VEGF signaling to mimic the endogenous regulation. As shown in Fig. 3a, the addition of VEGFa led to a rapid upregulation of p-ERK-1/2 (Thr202/Tyr204) and p-AKT (Ser473) in NC-shRNA transfected control cells. Strikingly, both of these responses to VEGFa stimulation were markedly reduced in SEC14L2 shRNA transfected cells (Fig. 3a-c). Additionally, in human umbilical arterial endothelial cells (HUAECs) with SEC14L2 knockdown, the p-AKT level was also inhibited significantly, whereas the p-ERK level was not changed a lot (Supplementary Fig. 7). In line with the compromised VEGF signaling in HUVECs, depletion of sec14l3 also caused a reduction of p-Erk and p-Akt in zebrafish embryos (Fig. 3d, e). These results indicate that Sec1413/SEC14L2 positively regulate VEGF signaling pathway.

Next, we tried to rescue the vasculogenic defects (i.e., narrow $\mathrm{DA}$ and PCV) in sec14l3 morphants by injecting $c a-M E K$ and/or ca-AKT mRNAs encoding the constitutively active (ca) forms of human MEK and AKT respectively ${ }^{38,39}$. To our surprise, $50 \mathrm{pg}$ ca-MEK mRNA injection alone only restored the DA formation in sec14l3 morphants, while $50 \mathrm{pg} c a-A K T$ mRNA only rescued $\mathrm{PCV}$. When the two mRNA species were co-injected, both DA and PCV of morphants were largely restored (Fig. 3f, g). These data provide strong evidence that sec14l3 acts through VEGF signaling to regulate vascular formation.

SEC14L2 promotes VEGFR2 phosphorylation at $\mathrm{Y}^{1175}$ site. Upon VEGFa stimulation, VEGFR2 molecules on the plasma membrane undergo dimerization and phosphorylation at several key tyrosine residues including the $\mathrm{Y}^{1054 / 1059}$ and $\mathrm{Y}^{1175}$ sites, which are essential for VEGF signaling to activate its downstream PLC $\gamma / E R K$ and PI3K/AKT pathways ${ }^{40}$. The finding that SEC14L2 knockdown reduces ERK and AKT activation (Fig. 3a-e, and Supplementary Fig. 7) prompted us to examine phosphorylation levels of VEGFR2 at both $\mathrm{Y}^{1054 / 1059}$ and $\mathrm{Y}^{1175}$ sites. Results showed that SEC14L2 knockdown had no significant effect on VEGFR2 $\mathrm{Y}^{1054 / 1059}$ phosphorylation (Fig. 4a, b). In contrast, $\mathrm{Y}^{1175}$ phosphorylation level showed a pronounced reduction in SEC14L2 shRNA cells (Fig. 4a, c), which is consistent with the reduction of activated ERK and AKT by SEC14L2 knockdown (Fig. 3a-c). To confirm functional conservation between zebrafish Sec14l3 and human SEC14L2 in VEGF signal transduction, a Flag-tagged full-length form of zebrafish Sec14l3 was transfected into SEC14L2 knockdown stable HEK293T cells with VEGFR2 overexpression. As a result, sec14l3 overexpression not only restored but even enhanced VEGFR2-Y'1175 phosphorylation following VEGFa stimulation (Fig. 4d, e). To test whether this regulation is specific, cytoplasmic regions of three different VEGF receptors, VEGFR1-cyto, VEGFR2-cyto, and VEGFR3-cyto, were overexpressed with Sec1413 for co-IP experiments respectively in HEK293T cells. Results showed that only VEGFR2-cyto could strongly interact with Sec14l3 (Fig. 5a), indicating that Sec14l3somehow specifically regulates VEGFR2, but not VEGFR1 or VEGFR3 signaling. Taken together, these data indicate that zebrafish Sec1413 is the counterpart of human SEC14L2 and both of them enhance VEGF signaling via regulating VEGFR2-Y1175 phosphorylation specifically.

Previous studies have demonstrated that decreased phosphorylation of VEGFR2-Y Y $^{1175}$ may be caused by prolonged exposure of the activated VEGFR2 to some tyrosine phosphatases beneath or on the plasma membrane. PTP1B has been reported and identified as such a specific phosphatase $\mathrm{e}^{21,22,41,42}$. To prove this notion, we compared the interaction between PTP1B and VEGFR2-cyto with or without SEC14L2 in HEK293T cells. The co-IP result showed that SEC14L2/Sec1413 could indeed prevent VEGFR2 from interacting with PTP1B (Fig. 5b). Then, we wondered whether knockdown of $P T P 1 B$ could reverse the effect of SEC14L2 depletion on VEGFR2-Y1175 phosphorylation. Strikingly, co-knockdown of PTP1B and SEC14L2 in HEK293T cells resulted in much higher phosphorylation levels of VEGFR2-Y1175 compared to SEC14L2 knockdown alone (Fig. 5c, d).

Consistent with these in vitro results, overexpression of VEGFR2-cyto mRNA or co-knockdown of $p t p 1 b$ in sec14l3 morphants led to a relatively normal lumen size of DA and PCV at 25 and $30 \mathrm{hpf}$ (Fig. 5e-h), while overexpression of VEGFR2cyto mRNA or knockdown of ptplb alone had no obvious effect on the DA and PCV formation (Supplementary Figs. 8, 9). Taken together, these in vitro and in vivo observations imply that SEC14L2/Sec14l3 may somehow protect VEGFR2-Y1175 phosphorylation from its specific phosphatase PTP1B and then stimulate VEGF signaling.

VEGFR2 trafficking is suppressed after SEC14L2 depletion. Based on the above results, in particular, the vesicle-like colocalization of SEC14L2 and VEGFR2 in HUVECs (Fig. 1d), we hypothesize that Sec1413/SEC14L2 may contribute to intracellular trafficking of VEGFR2. To address this issue, we performed surface biotinylation assay to detect internalization and recycling of overexpressed VEGFR2 in HEK293T cells (Supplementary Fig. 10). We found that, before induction of internalization, the biotin-labeled VEGFR2 protein level on the cell surface in SEC14L2 shRNA transfected HEK293T cells was comparable 
a

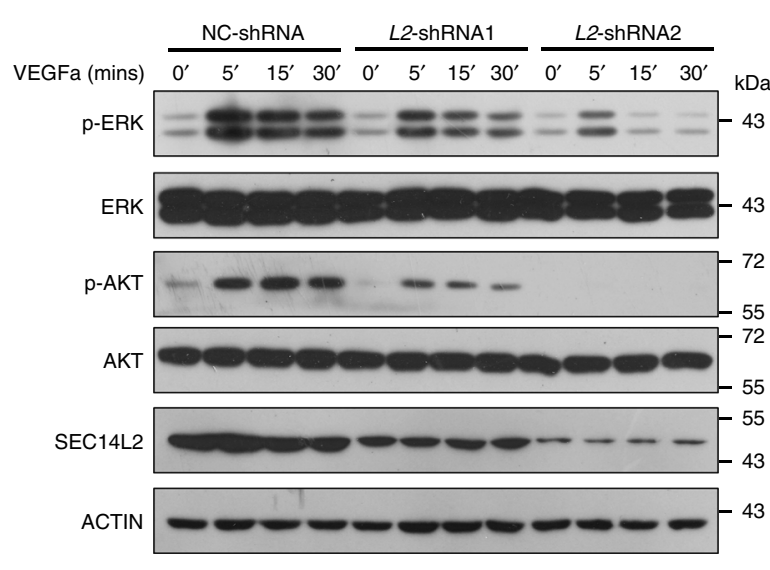

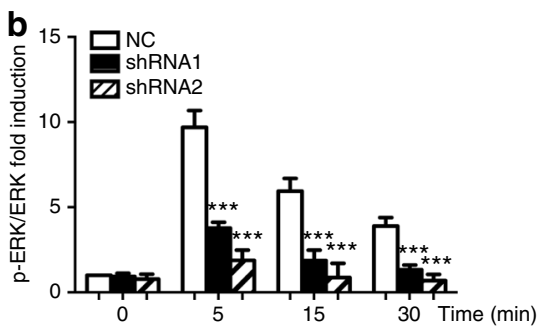

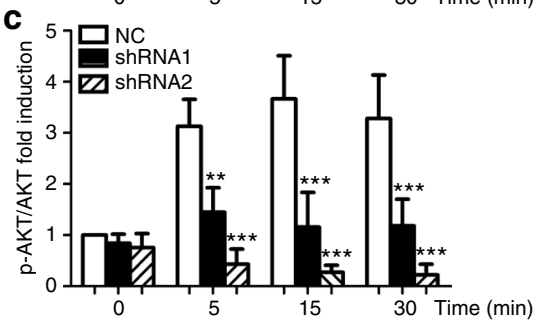

d

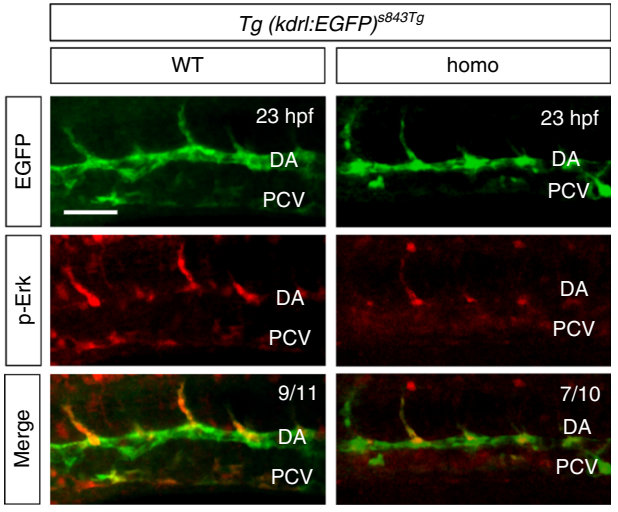

f
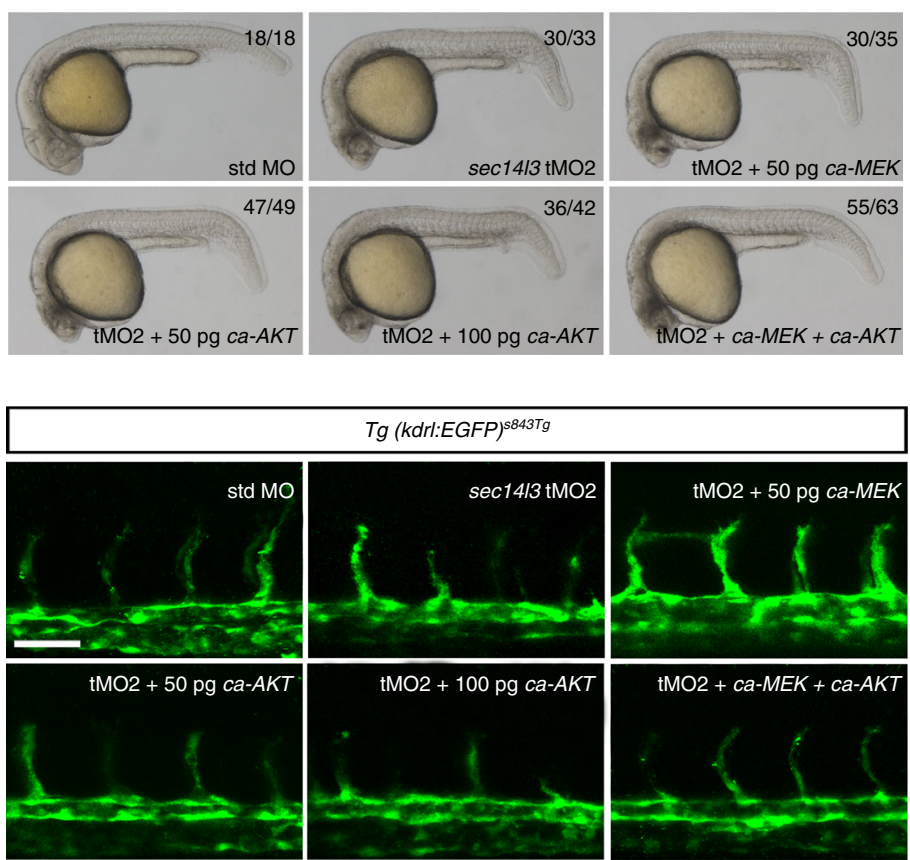

$\operatorname{Tg}(k d r l: E G F P)^{s 843 T g}$
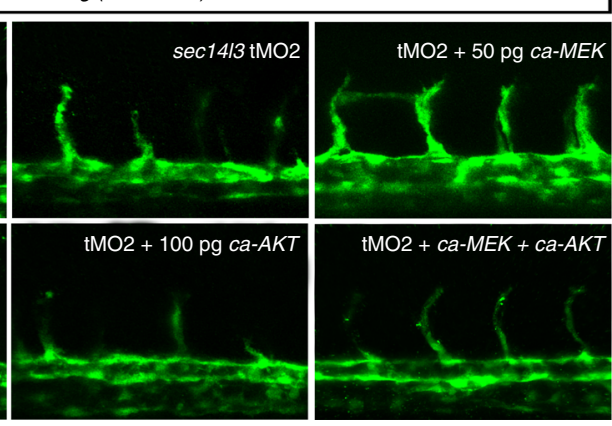

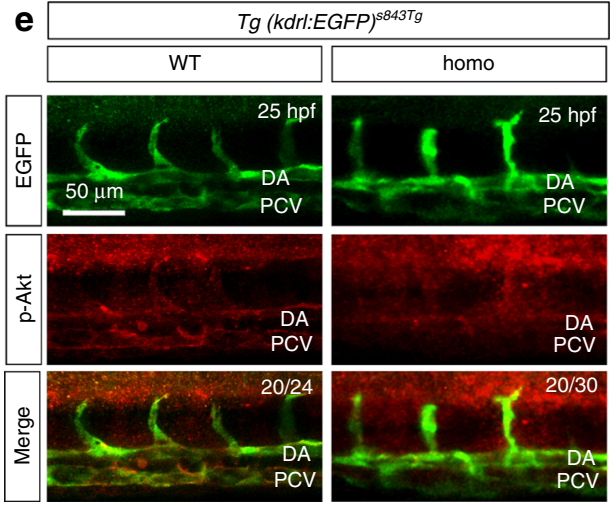

g

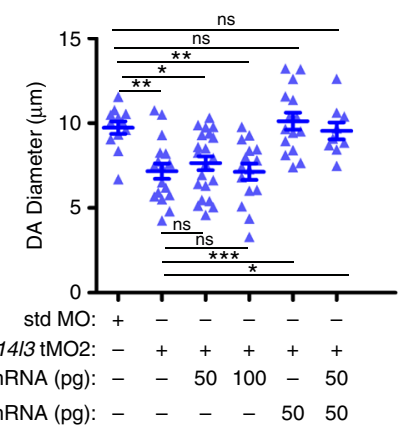

ca-MEKMRNA (pg): - $\quad-\quad-5050$

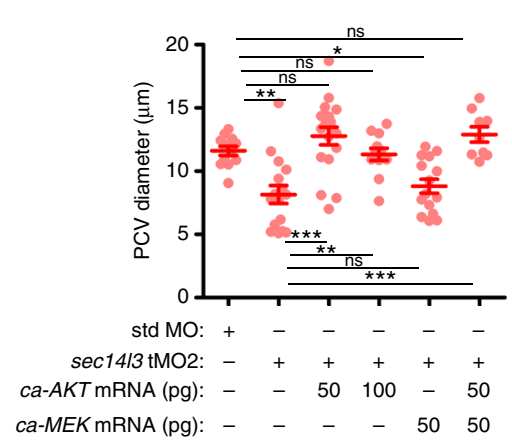

to that in control cells (Fig. 6a, the first lane and fifth lane); following induction of internalization, intracellular biotinlabeled VEGFR2 proteins gradually increased in the control cells, but this increase was much slower in SEC14L2 shRNA cells (Fig. 6a). Thus, SEC14L2 is a potential regulator of VEGFR2 trafficking.
Next, the transport of VEGFR2-containing endosomes in HUVECs was examined by immunostaining assay. Before induction of internalization, endogenous VEGFR2 proteins on the cell surface were labeled similarly in control and SEC14L2 shRNA transfected cells (Fig. $6 \mathrm{~b}$, the first panel). After internalization induction at $37^{\circ} \mathrm{C}$ for $10 \mathrm{~min}$, the 
Fig. 3 SEC14L2/sec14/3 knockdown attenuates VEGF signaling. a SEC14L2 knockdown counteracts VEGFa-motivated p-ERK and p-AKT levels in HUVECs. HUVECs were infected with NC or SEC14L2 shRNA for $48 \mathrm{~h}$. After VEGFa stimulation for 5, 15 or 30 min, cell lysates were harvested and immunoblotted with indicated antibodies. b, c Statistical results of relative p-ERK (b) and p-AKT (c) levels in $\mathbf{a}$. The grey intensity of each band was measured for calculating the ratio of $p$-ERK to ERK (b) and p-AKT to AKT (c). Data are then normalized to control group with $0^{\prime}$ stimulation and represented as mean \pm SEM from three independent experiments. d-e Depletion of sec14/3 decreases p-Erk (d) and p-Akt (e) levels in zebrafish embryos. Embryos from intercrossing sec14/3 heterzygous in Tg(kdrl: GFP) $843 \mathrm{Tg}$ transgenic background were harvested at $23 \mathrm{hpf}$ for p-Erk antibody immunostaining (d) or at $25 \mathrm{hpf}$ for p-Akt antibody staining (e). DA, dorsal aorta. The ratio in the right corner indicates the number of embryos with reduced staining/the number of observed embryos. Scale bars, $50 \mu \mathrm{m}$. f The defective DA/PCV lumen formation in sec14/3 morphants can be partially rescued by ca-MEK or ca-AKT mRNA injection. Five nanograms std-MO or sec14l3-tMO2 in combination with 50 or 100 pg ca-MEK or ca-AKT mRNA was injected into one-cell stage embryos. Their morphology (the upper panel) or vasculature defects (the bottom panel) at $25 \mathrm{hpf}$ are separately shown. The ratio in the right corner indicates the number of embryos with indicated morphology/the number of observed embryos. Scale bars, $50 \mu \mathrm{m}$. $\mathbf{g}$ Statistic results of the DA (upper panel) or PCV (bottom panel) luminal diameters of each group at $30 \mathrm{hpf}$. Twenty embryos of each group from one representative experiment were calculated and shown here. To quantify the DA and PCV luminal diameter for an embryo, five different vessel regions of the same fish were measured to calculate the mean value, which was used to represent its vessel diameter of this fish. Three independent experiments were carried out. ${ }^{\star} p<0.05 ;{ }^{\star \star} p<0.01$; ${ }^{\star \star \star} p<0.001 ; \mathrm{ns}$, not significant. ANOVA tests were used for statistical analyses. Source data are provided as a Source Data file

a

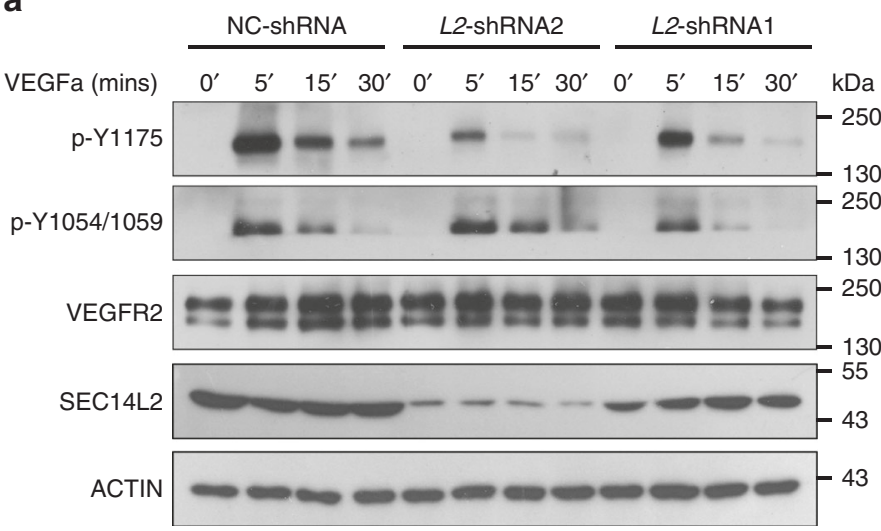

d

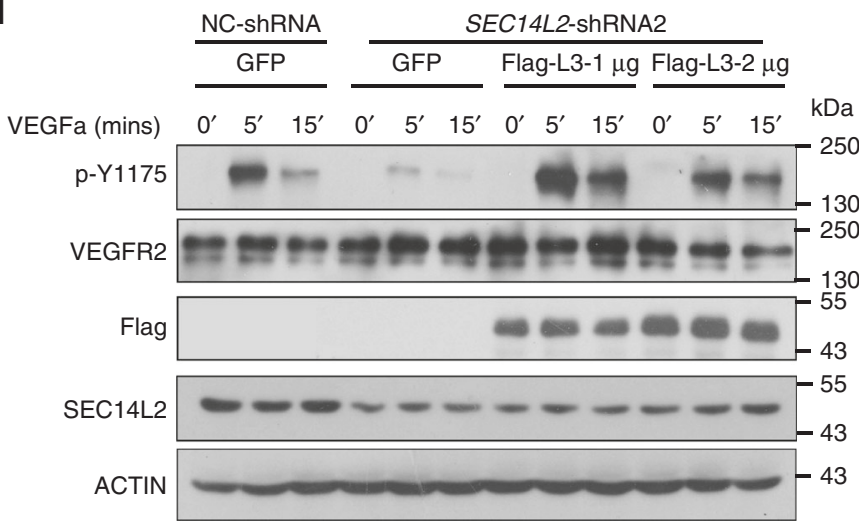

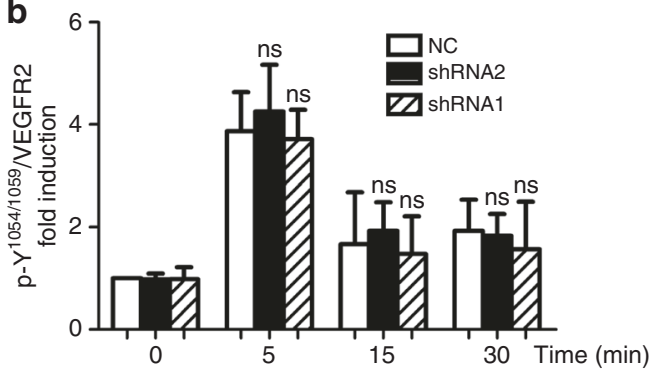
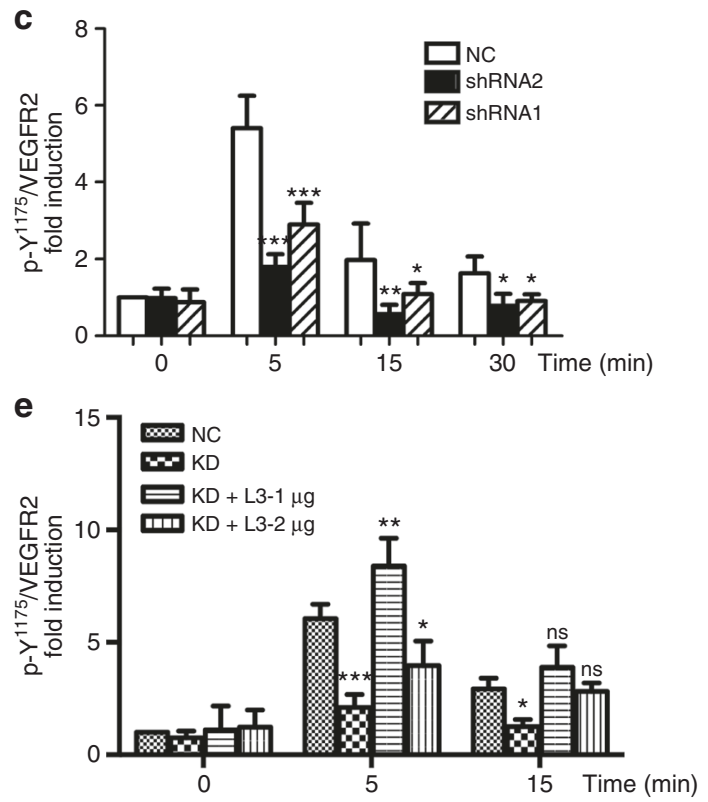

Fig. 4 SEC14L2 knockdown decreased VEGFR2 phosphorylation at Y1175 site. a Western blot analysis of cell lysates from NC or SEC14L2 shRNA infected HUVECs. Cells were infected and starved overnight, followed by $100 \mathrm{ng} \mathrm{ml}^{-1}$ VEGFa stimulation for 5,15 or 30 min, respectively. VEGFR2 ( $p$-Y1175), VEGFR2 ( $p-Y^{1054 / 1059}$ ), and total VEGFR2 levels were examined by immunoblotting using respective antibodies. b, c Statistical results of $p-V E G F R 2-Y^{1054}$ / 1059 (b) and p-VEGFR2-Y1175 (c) levels in a relative to total VEGFR2. Data are normalized to control group without stimulation and shown as mean \pm SEM from three independent experiments. ${ }^{\star} p<0.05 ;{ }^{\star \star} p<0.01 ;{ }^{\star \star \star} p<0.001$; ns, not significant. $\mathbf{d}$, e Overexpression of zebrafish sec14/3 in HEK293T cells restores the level of $p$-VEGFR2-Y1175 inhibited by SEC14L2 knockdown. One or two micrograms sec1413 plasmid was transfected into SEC14L2 knockdown stable HEK293T cells with VEGFR2 overexpression. Following starvation overnight, $100 \mathrm{ng} \mathrm{ml}^{-1}$ VEGFa was added and stimulation continues for 0 , 5 or 15 min. Quantification data of the relative p-VEGFR2-Y1175 level from three repeated experiments are normalized to control group with $0^{\prime}$ stimulation and shown on the right $(\mathbf{e}) .{ }^{\star} p<0.05 ;{ }^{\star \star} p<0.01 ;{ }^{\star \star \star} p<0.001$; ns, not significant. The loading volume of $\mathbf{a}, \mathbf{d}$ was adjusted for the same amount of total VEGFR2 level in each lane. Two-way ANOVA tests were used for statistical analyses in $\mathbf{b}, \mathbf{c}$, and $\mathbf{e}$. Source data are provided as a Source Data file 
a $\begin{array}{rcccccc}\text { 3XFlag-Sec1413 } & + & - & + & + & + & \\ \text { Myc-VEGFR-Cyto } & - & \text { VR1 } & \text { VR1 } & \text { VR2 } & \text { VR3 } & \text { KDa }\end{array}$

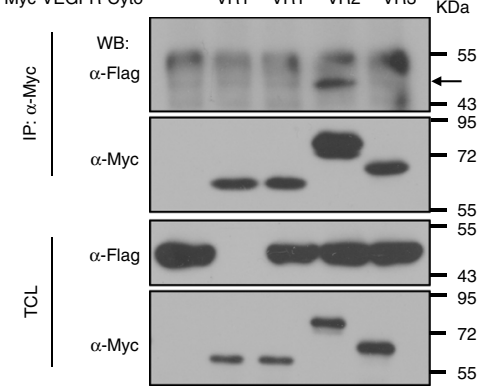

C
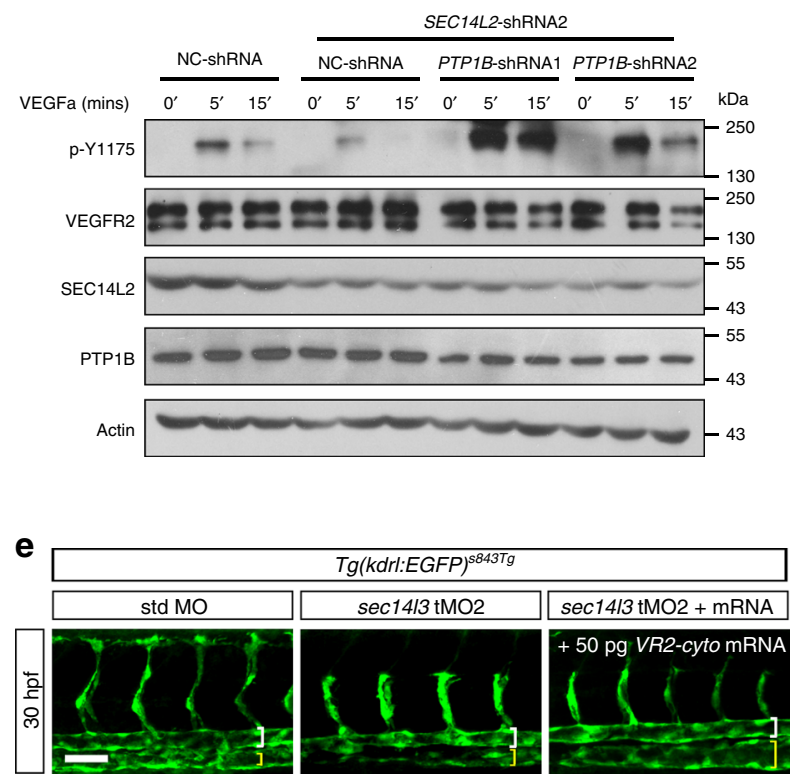

g
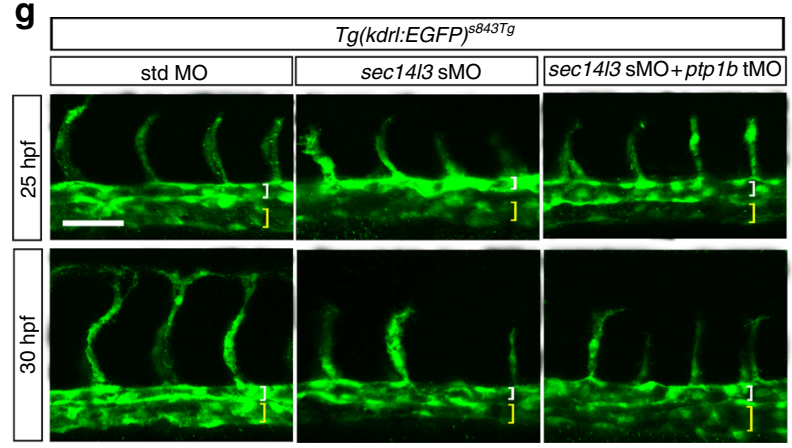

VEGFR2-containing endosomes in SEC14L2 knockdown HUVECs were significantly decreased compared to the control cells (Fig. 6b, c). Additionally, co-localization analysis between internalized VEGFR2 and the early endosomal marker EEA1 showed that SEC14L2 shRNA cells contained far fewer VEGFR2/EEA1 double positive endosomes than control cells (Fig. 6b, c), suggesting a requirement of SEC14L2 for VEGFR2 internalization into early endosomes.

It has been reported that in resting endothelial cells, VEGFR2 displays both the cell surface distribution and internal vesicular pool storage, which can be redistributed to the cell periphery upon VEGF stimulation ${ }^{12}$. To investigate whether SEC14L2 b
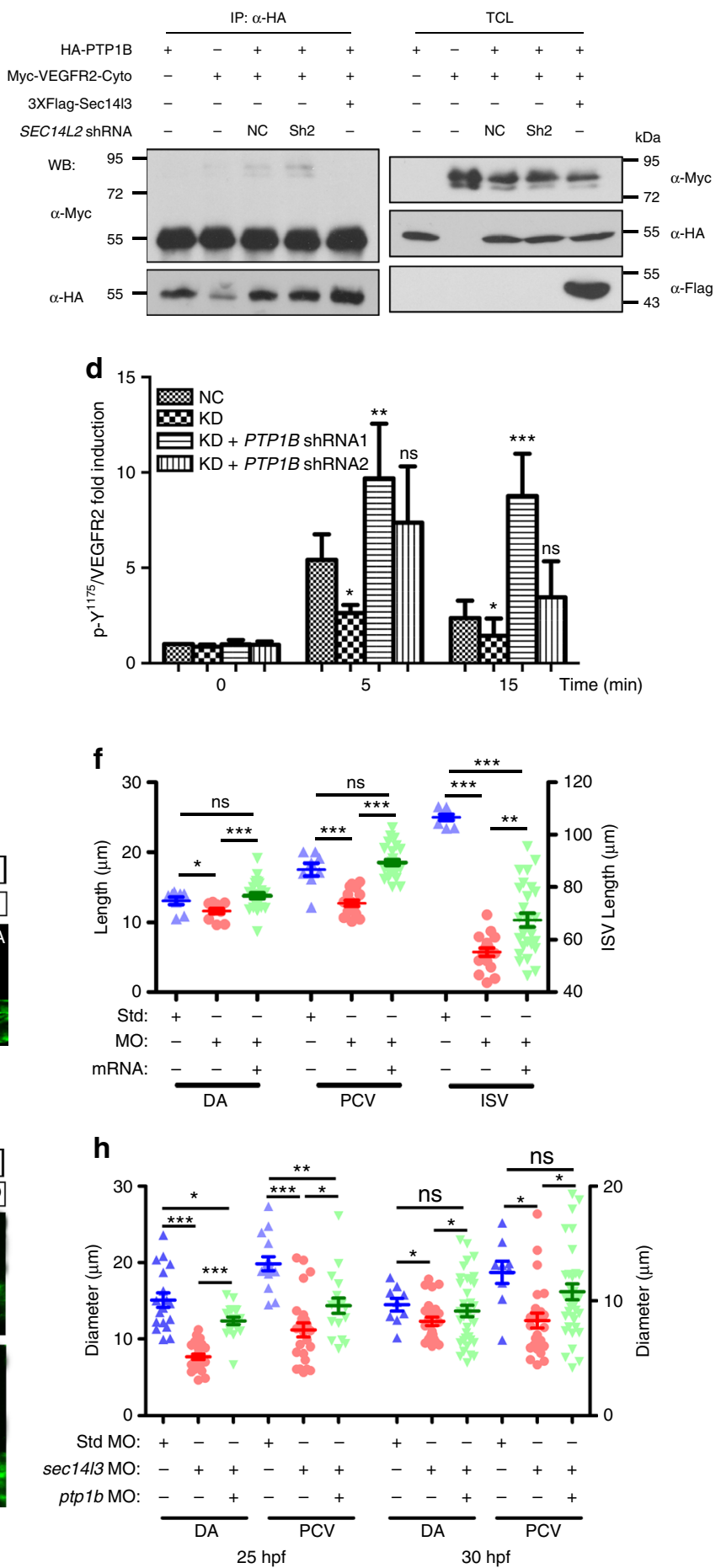

participates in this process, we performed surface biotinylation assay over a 35 -min time course $\left(20 \mathrm{~min}\right.$ internalization at $22^{\circ} \mathrm{C}$ followed by $15 \mathrm{~min}$ recycling at $37^{\circ} \mathrm{C}$ ). In NC-shRNA control cells, about $40 \%$ of internalized VEGFR 2 could be recycled back to the plasma membrane and this recycling rate was enhanced to approximately $80 \%$ in the present of VEGFa stimulation (Fig. 6d), which is similar to the previous report ${ }^{12,22}$. However, knocking down SEC14L2 drastically decreased the VEGFR2 recycling rate to about 8 and $20 \%$, respectively, with or without VEGFa stimulation (Fig. 6d). Therefore, we conclude that SEC14L2 may promote VEGFR2 intracellular mobilization at least in two ways, internalization from and recycling back to the cell surface. 
Fig. 5 Enhanced VEGFR2-Y1175 phosphorylation restores SEC14L2/sec14/3 depletion effects. a Sec14I3 specifically interacts with the cytoplasmic region of VEGFR2 in HEK293T cells. The cytoplasmic region of VEGFR1, VEGFR2 or VEGFR3 was individually transfected with Flag-tagged Sec14I3 into HEK293T cells for immunoprecipitation (IP) and immunoblotting. TCL, total cell lysate. b SEC14L2/Sec14I3 could alleviate the interaction between PTP1B and the VEGFR2-cyto in HEK293T cells. HA-tagged PTP1B and Myc-tagged VEGFR2-cyto were co-transfected into HEK293T cells with Flag-tagged Sec14l3 or SEC14L2 shRNA. After $72 \mathrm{hpf}$, cells were harvested and lysed for immunoprecipitation assay using an HA antibody. WB, western blot. c, d PTP1B knockdown rescues p-VEGFR2-Y1175 level inhibited by SEC14L2 knockdown in HEK293T cells. PTP1B shRNA1 or shRNA2 was transfected into SEC14L2 shRNA stable HEK293T cells with VEGFR2 overexpression. p-VEGFR2-Y1175 levels were checked after $100 \mathrm{ng} \mathrm{ml}^{-1}$ VEGFa stimulation for 0,5 or 15 min. The loading volume was adjusted for the same amount of total VEGFR2 level in each lane. Quantification data of the relative p-VEGFR2-Y1175 level from three independent experiments are shown in $\mathbf{d}(n=3)$. e The cytoplasmic region of VEGFR2 mRNA rescues luminal defects in sec14/3 morphants. Five nanograms std-MO or sec14I3-tMO2 in combination with 50 pg VEGFR2-cyto mRNA was injected into one-cell stage embryos from Tg(kdrl: GFP)s843Tg transgenic fish, and harvested at $30 \mathrm{hpf}$ for the measurement of luminal diameters /ISV length. f Statistical result of the luminal diameters of DA, PCV, and ISV in e. 20 embryos of each group from one representative experiment were calculated and shown here. Three independent experiments were carried out. g, h ptp 16 knockdown partially restores the lumen size of DA/PCV in zebrafish sec14/3 morphants. $T g(k d r l: G F P)$ s843Tg transgenic embryos were injected with $0.5 \mathrm{ng}$ std-MO or sec14/3-sMO in combination with $5 \mathrm{ng}$ ptp1b-tMO and harvested at 25 and 30 hpf for the measurement of luminal diameters. Twenty embryos of each group were calculated, and statistical data from three independent experiments are shown in $\mathbf{h} .{ }^{\star} p<0.05$; ${ }^{\star \star} p<0.01$; ${ }^{\star \star \star} p<0.001$; ns, not significant. ANOVA tests were used for statistical analyses in $\mathbf{d}, \mathbf{f}$, and $\mathbf{h}$. Scale bars, $50 \mu \mathrm{m}$. Source data are provided as a Source Data file

Furthermore, we tried to figure out the final destination of VEGFR2 after knocking down SEC14L2 in HUVECs. Western blotting results showed that the total VEGFR2 protein level is not significantly affected when SEC14L2 is depleted, exclusive of its possible role in VEGFR2 degradation (Supplementary Fig. 11). When co-staining VEGFR2 with late endosome markers, RAB7 or CD63, we found that SEC14L2 knockdown led to more VEGFR2 accumulation in these compartments (Fig. 6e, f). Taken these results together, we would like to prospect that more VEGFR2 is stored in late endosomes but not delivered to the lysosome for degradation in SEC14L2 deficient cells, which is consistent with previous reports ${ }^{12}$.

Sec1413/SEC14L2 physically interact with VEGFR2 and RABs. We next set out to investigate how Sec14l3/SEC14L2 mediate VEGFR2 trafficking. Given that Sec14l3 interacts with VEGFR2cyto specifically (Fig. 5a) and SEC14L2 co-localizes with VEGFR2 endogenously in vesicular compartments (Fig. 1d), we first demonstrated that endogenous SEC14L2 indeed interacts with endogenous VEGFR2 and this interaction could be strengthened upon VEGFa stimulation (Fig. 7a), indicating that SEC14L2 prefers binding to activated VEGFR2. To further prove this point, wild-type form (WT-VEGFR2) or kinase mutation form (KM-VEGFR2) of VEGFR2 was co-transfected with Flag-tagged zebrafish Sec1413 in HEK293T cells for Co-IP experiments ${ }^{43}$. We found that Sec1413 associates with WT-VEGFR2 much stronger than KM-VEGFR2 (Fig. 7b, c). Taking these data together, we conclude that Sec1413 favors associating with activated VEGFR2. Additionally, domain mapping results revealed that the Sec14 domain of Sec14l3 was essential for its interaction with VEGFR2 (Fig. 7d, e).

As a member of the receptor tyrosine kinases (RTKs) family, VEGFR2 is regulated by various Rab proteins at the intracellular trafficking level, which renders vesicle transport to particular destination somehow through recruiting their corresponding effectors $^{44}$. Considering that Sec1413/SEC14L2 act during VEGFR2 trafficking, we wondered whether Sec14l3 could associate with $\mathrm{RAB}$ family proteins. Four members of RAB proteins, representing distinct endocytosis routes ${ }^{45-47}$, were selected for co-IP experiments in HEK293T cells. Results revealed that only RAB4A and RAB5A could strongly interact with Sec14l3 (Fig. 7f), which coincides with the function of Sec14l3 in VEGFR2 internalization and recycling (Fig. 6). Furthermore, these interactions were confirmed by endogenous co-IP data in HUVECs (Fig. 7g, h). Domain mapping analyses disclosed that the N-terminal CRAL-TRIO domain and the C-terminal GOLD2 domain of Sec1413 were required for interaction with RAB4A
(Fig. 7d, i). Therefore, Sec1413 may promote VEGFR2 endocytosis and recycling through its interaction with RAB5A and RAB4A.

Sec1413/SEC14L2 are required for RAB5A/4A activation. Like other GTPases, the RAB5A/4A cycle between an active (GTPbound) and an inactive (GDP-bound) state, which is of critical importance to modulate vesicle trafficking processes ${ }^{48,49}$. We wonder whether Sec1413/SEC14L2 are required for the activity of RAB5A/4A to regulate VEGFR2 trafficking. To this end, we first compared the association of Sec14l3 with RAB5A/4A-GDP and RAB5A/4A-GTP. Transfection and in vitro binding assays revealed that Sec14l3 preferred binding to the GDP-bound state of RAB5A/4A (Fig. 8a, b). To further investigate the function of Sec1413/SEC14L2 in RAB5A/4A regulation, we performed immunoprecipitation assay using a specific anti-RAB5A/4A-GTP antibody or pull-down assay using purified GST-R5BD, which specifically binds to RAB5A-GTP ${ }^{50}$. Compared to the NC-shRNA control, knocking down SEC14L2 caused more than 50\% reduction of the RAB5A-GTP and RAB4A-GTP amount (Fig. 8c, d), which is consistent with the compromised VEGFR2 internalization and recycling processes (Fig. 6). Taken together, these results suggest that Sec1413/SEC14L2 facilitate the formation of RAB5A/ $4 \mathrm{~A}$ in their GTP-bound active states, and then probably accelerate the movement of VEGFR2 vesicles to early endosomes and recycling endosomes to enhance VEGF signaling (Fig. 8e).

\section{Discussion}

Given the critical roles of endocytosis in VEGFR2 signaling, numerous relevant factors that have effects on receptor trafficking along endosomes have been identified, and most of them were demonstrated to participate in VEGFR2 internalization or degradation 16,22,23,41,51-54. However, few factors were found to regulate a relatively complete VEGFR2 intracellular trafficking process, such as receptors internalization from and recycling back to the plasma membrane for enhancing signaling output. Here, we propose that the lipid binding proteins Sec1413/SEC14L2 concurrently provoke VEGFR2 uptake from the plasma membrane and return from the endosomal pool to maximize signaling strength upon ligand stimulation. Mechanistically, Sec14l3/ SEC14L2 conjugate activated VEGFR2 into RAB5-positive sorting endosomes and RAB4-positive fast recycling endosomes through their interaction to protect VEGFR2 from dephosphorylation by the peri-membrane tyrosine phosphatase PTP1B (Fig. 8e). Therefore, Sec1413/SEC14L2 deficiency disturbs VEGFR2 internalization and recycling, leading to a prolonged exposure to PTP1B and subsequent compromises of PLC $\gamma /$ ERK and PI3K/AKT activation, which consequently results in defective 
a
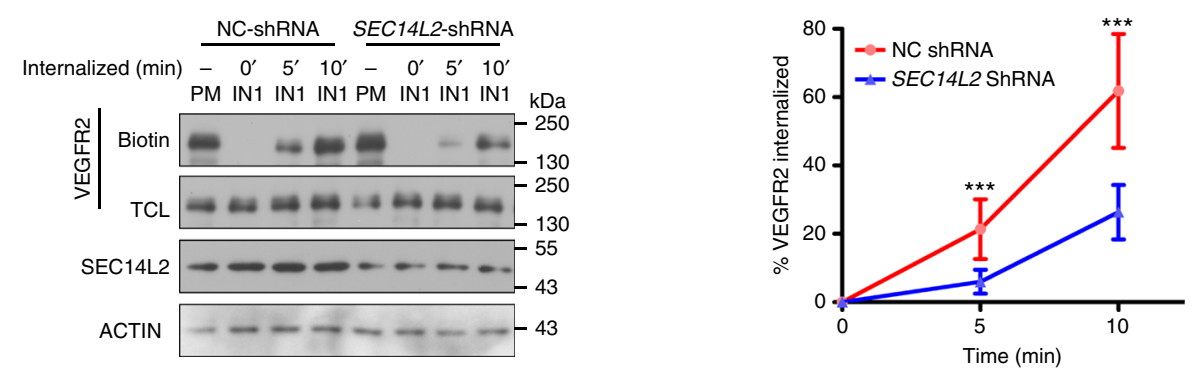

b

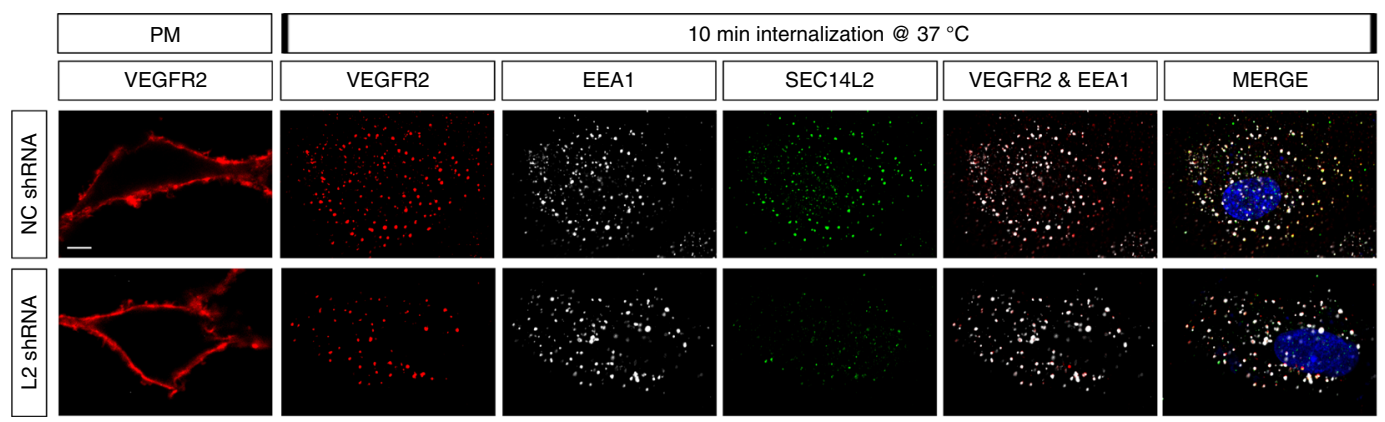

C
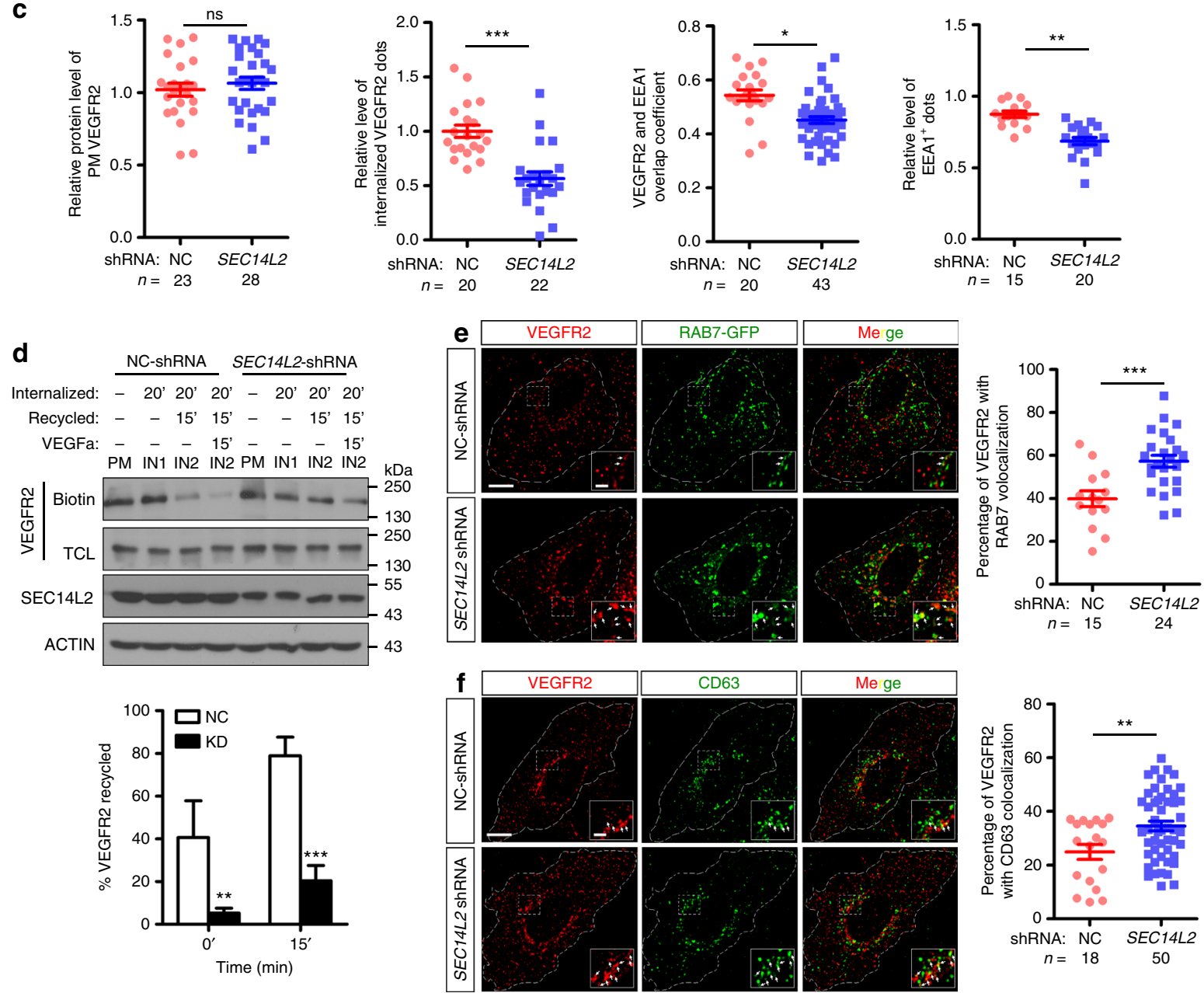

vasculature morphogenesis in zebrafish embryos in vivo and vascular tubulogenesis in vitro. The effective induction of VEGF signaling through overexpression of cytoplasmic region of VEGFR2 mRNA or suppression of $p t p 1 b$ expression or overexpression of constitutively active $M E K / A K T$ partially restored the narrowed DA and PCV lumens in zebrafish sec14l3 morphants. This study provides insight into the regulation of VEGFR2 endocytosis in vertebrate vascular system formation.

It was apparent that $\sec 14 l 3$ inactivation specifically affected vasculogenesis, whereas the angiogenic process appeared to be nearly intact, although both processes heavily rely on VEGF signaling. The pattern of how sec14l3 is spatiotemporally 
Fig. 6 SEC14L2 knockdown disturbs VEGFR2 internalization and recycling processes. a Western blotting results show impairment of biotinylated VEGFR2 internalization in SEC14L2 knockdown cells. VEGFR2-overexpressing HEK293T cells were transfected with NC or SEC14L2 shRNA for internalization assay described as supplementary Fig. 10. Quantified internalized VEGFR2 levels are shown on the right as mean \pm SD from three independent experiments $(n=3)$. Red and blue lines represent control and SEC14L2 shRNA transfected group, respectively. TCL, total cell lysate. ${ }^{\star \star \star} p<0.001$. b, c Analysis of VEGFR2 internalization based on immunostaining assay in HUVECs. HUVEC cells infected with NC or SEC14L2 shRNA were cultured for internalization assay and then cells were fixed for immunostaining with anti-EEA1 (white) and anti-SEC14L2 (green) antibodies. Quantification data of plasma membrane VEGFR2 (red) before internalization, internalized VEGFR2 after internalization, overlapping coefficient between internalized VEGFR2 and EEA1 $(n=40$ cells) as well as EEA1 positive dots are shown individually in $\mathbf{c} .{ }^{\star} p<0.05 ;{ }^{\star \star} p<0.01$; ${ }^{\star \star \star} p<0.001$; ns, not significant. d Western blot analysis of VEGFR2 recycling over a 35 min time course. VEGFR2-overexpressing HEK293T cells were transfected with NC- or SEC14L2 shRNA for $48 \mathrm{~h}$ and used for recycling assay as detailed in Supplementary Fig. 10. The recycling rate of VEGFR2 was measured with or without $100 \mathrm{ng} \mathrm{ml}^{-1} \mathrm{VEGFa}$ stimulation. Quantification data are shown as mean \pm SD from three independent experiments on the right $(n=3)$. ${ }^{\star \star} p<0.01$; ${ }^{\star \star} p<0.001$. e, f SEC14L2 knockdown promotes VEGFR2 localization in $\mathrm{RAB7}^{+}(\mathbf{e})$ or $\mathrm{CD}^{+} 3^{+}(\mathbf{f})$ compartments. HUVEC cells infected with NC or SEC14L2 shRNA were transfected with RAB7-EGFP (e) or harvested directly (f) for anti-VEGFR2 (red) and EGFP/CD63 (green) staining. Regions in the boxes are enlarged in the right corner. Scale bars, $10 \mu \mathrm{m}$. The percentage of VEGFR2 co-localized with RAB7/CD63 is shown individually in the right, $n$ indicates observed cell number. ${ }^{\star \star} p<0.01 ;{ }^{\star \star *} p<0.001$. Two-way ANOVA tests were used for $\mathbf{a}, \mathbf{d}$ and $t$-tests for $\mathbf{c}$, e, and $\mathbf{f}$. Source data are provided as a Source Data file

expressed may provide an explanation for this. Before the onset of the major axial vessels at $19 \mathrm{hpf}$, sec14l3 is expressed in both arterial and venous progenitors. And thereafter, it is gradually restricted into the PCV compartment, not DA or ISVs. This particular time window is corresponding to vasculogenesis, but not angiogenesis context during vascular morphogenesis. We did notice a reduced p-Erk level in DA of sec14l3 deficient embryos yet with the robust signal in selected endothelial cells, which might be enough for the tip cell selection and subsequent ISVs formation. This notion is compatible with ISV defects in $k d r l$ and plc 1 mutant embryos, which are caused by a loss of pErkpositive sprouting ISV endothelial cells in dorsal aorta ${ }^{55}$.

Inhibition between PI3K/AKT and PLC $\gamma /$ ERK signaling has been well documented ${ }^{38,56}$. Within the arterial-fated angioblast, VEGFR2 signaling induces PLC $\gamma /$ ERK kinase cascade and subsequently stimulates expression of several genes in the Notch pathway to ensure the arterial cell fate. It has been reported that overexpression of constitutively active MEK or treatment with agonists of PLC $\gamma /$ ERK signaling can rescue the DA formation in zebrafish embryos lacking vegf $a^{55,57-60}$. On the other hand, in the venous-fated angioblast, PI3K/AKT signaling is activated to antagonize ERK activity, and overexpression of constitutively active Akt promotes the venous cell fate ${ }^{38}$. Therefore, it seems coincident with previous reports that overexpression of $c a-M E K$ or $c a-A K T$ could rescue the DA or PCV formation respectively in sec14l3 mutant embryos bearing a relatively low level of VEGF signaling. Since both PLC $\gamma /$ ERK and PI3K/AKT pathways are downstream branches of the VEGF receptor, how they are preferentially activated in different endothelial progenitor cells or at different developmental stages remain intriguing questions. Furthermore, how Sec1413 exactly regulates these processes also need to be investigated in the future.

Upon VEGF ligands stimulation, VEGFR2 is engaged in dimerization to induce phosphorylation of several tyrosine residues in its cytoplasmic domain. Meanwhile, various phosphatases have been reported to remove tyrosine phosphorylation at distinct sites ${ }^{21,61-63}$. Given that PTP1B specifically dephosphorylates VEGFR2 at the $\mathrm{Y}^{1175}$ site, we merely examined the rescue effect of PTP1B depletion following Sec14l3/SEC14L2 knockdown. However, we cannot exclude the possible involvements of other phosphatases, especially those transmembrane receptor-type PTPs, such as DEP1/CD148, VE-PTP and so on ${ }^{62,63}$. Knocking down these phosphatases respectively in Sec14l3/SEC14L2 deficient embryos may provide more information.

Besides VEGF ligands, PECAM-1 mediated fluid shear stress signaling could also recruit VEGFR2 and then phosphorylate it at $\mathrm{Y}^{1175}$ site along with VE-Cadherin, thus regulating vascular remodeling, vascular homeostasis, cardiac development and atherogenesis ${ }^{37}$. In this study, we didn't focus on this branch, largely due to our in vivo observations that $\sec 14 l 3$ specifically exists and functions during zebrafish vasculogenesis before the onset of blood flow. Additionally, different from mammalian vascular development, it has been reported that changes in shear stress appear to be relatively unimportant in the initial stages of zebrafish angiogenesis because blood vessels can be successfully formed in the absence of heartbeat and blood flow until 14 days postfertilization $^{64}$. Even so, we still cannot exclude that Sec1413 could participate in regulating fluid shear stress-induced VEGFR2 activation during later stages of zebrafish angiogenesis. It will be interesting to explore this possibility in the future.

Previous studies have shown that VEGFR2 endocytosis proceeds in a clathrin-dependent internalization, followed by the cascade of RAB recruitments to deliver VEGFR2 to the plasma membrane for recycling, into late endosome for storage, or into lysosome for degradation ${ }^{65}$. In this work, we demonstrate that Sec1413 could directly interact with RAB5A/4A and promote their GTP-bound states formation, and probably orchestrate VEGFR2 internalization and recycling back to the plasma membrane. After performing in vitro GEF assay based on mant-GTP $\gamma S$ fluorescence, we proposed that Sec14l3 could not act as a GEF to accelerate the GTP loading activity of RAB5 directly (Supplementary Fig. 12). Actually, whether Sec1413 could regulate the GTP hydrolysis activity of RAB5A/4A also need to be determined by MESG-based single-turnover assay. In addition, whether the intrinsic GTPase activity of Sec14l3 is required for the activation of RAB5A/4A also awaits further investigation. Based on our data here, we would like to propose the following potential mechanisms of SEC14L2/Sec1413 acting on RAB5A/4A activation. (1) Sec14l3 might serve as an adaptor to recruit a GEF protein for RAB5A/4A. Although we have not detected any interaction between Sec14l3 and several well-known GEF proteins of RAB5, such as Rabex5 and RABGEF1, we still cannot exclude this possibility, since there might be other unidentified GEFs for RAB5A/4A that could participate in this process. (2) Sec1413 belongs to atypical class III PITPs, which are implicated in the traffic of phosphoinositides (PIs) between different membrane compartments $^{24}$. Different PI species display distinct subcellular distributions where they play intriguing functions. For example, $\mathrm{PI}(3) P, \mathrm{PI}(4) P$, and $\mathrm{PI}(3,5) P_{2}$ are found predominantly within early, recycling and late endosomes, respectively ${ }^{66}$, which are essential for appropriate endosomal trafficking events. Drosophila PI 3-phosphatase MTMR13 promotes $\mathrm{PI}(3) P$ turnover at endosomes to activate Rab5 family member Rab21, PI(3)P levels in early endosome may drive RAB5-RAB7 conversion and so on 67,68 . Therefore, it deserves further exploration of whether Sec 1413 could also motivate specific phosphoinositides transportation along vesicles to regulate endosome dynamics, either in morphology or in effector recruitment. 
a

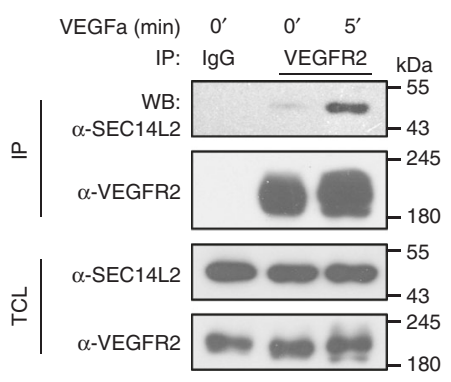

C

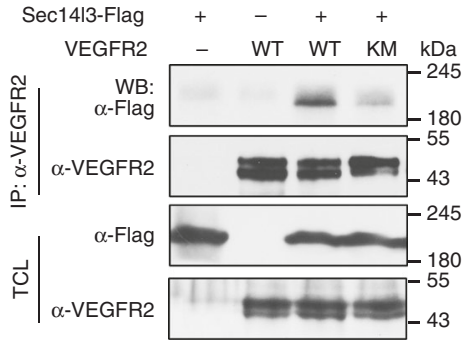

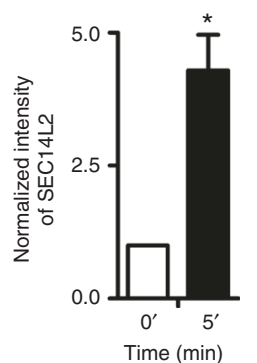

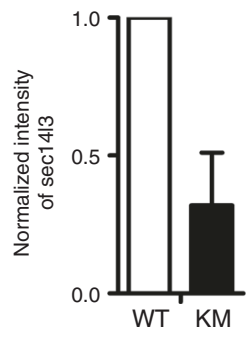

b

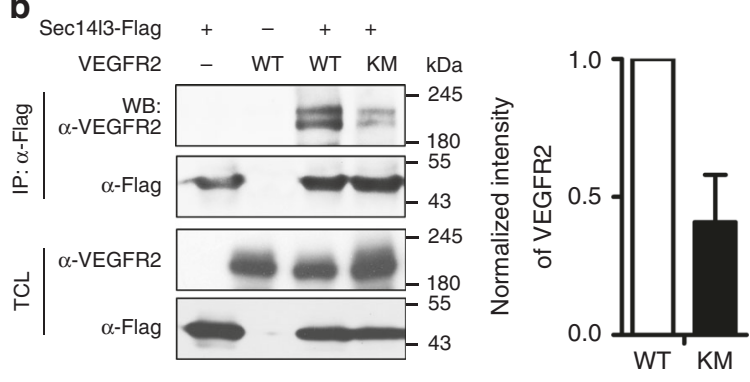

d

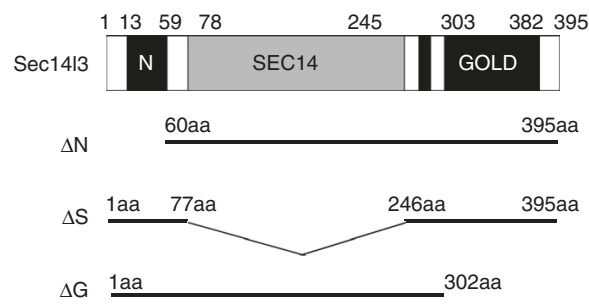

$\mathbf{f}$

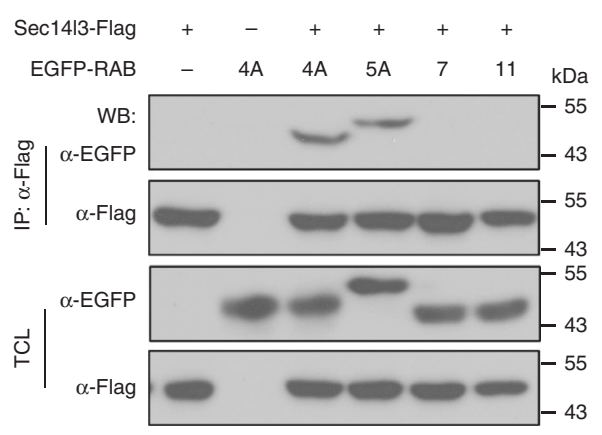

i

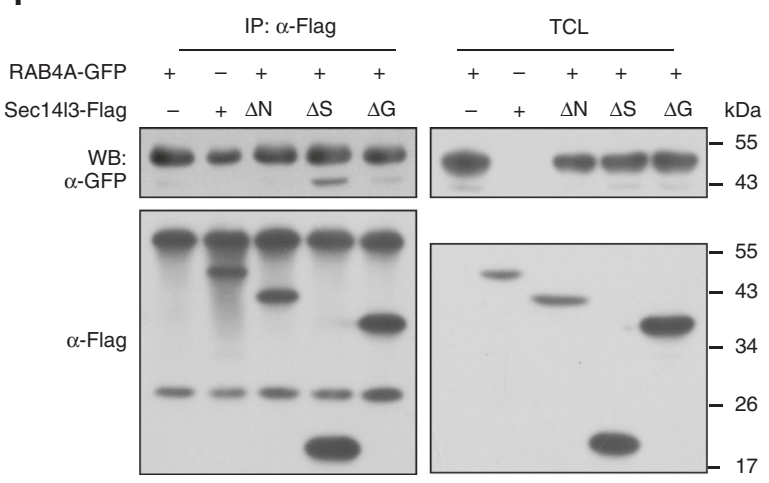

Fig. 7 Sec14I3/SEC14L2 interact with VEGFR2 and RAB4A/5 A via complementary domains. a SEC14L2 interacts with VEGFR2 endogenously in HUVECs. HUVEC cells were starved and stimulated with $100 \mathrm{ng} \mathrm{ml}^{-1}$ VEGFa for 5 min before harvest for IP and immunoblotting. TCL, total cell lysate. IgG serves as a negative control. Quantification of the interaction from three independent experiments is shown on the right $(n=3)$. ${ }^{*} p<0.05$. $\mathbf{b}, \mathbf{c}$ Sec14l3 shows a much stronger association with WT-VEGFR2 than its kinase domain mutation form (KM-VEGFR2). Different forms of VEGFR2 were co-expressed with Flag-tagged Sec14I3 in HEK293T cells. WB, western blot. Quantification of the interaction is shown on the right $(n=3)$. $t$-tests were used for statistical analyses in a-c. d Schematic diagrams of Sec14/3 and its truncated mutants. Numbers above the diagram indicate the corresponding amino acid positions. $\Delta \mathrm{N}$ indicates $\mathrm{N}$-terminal CARL-TRIO domain deletion; $\Delta \mathrm{S}$, Sec14 domain deletion; $\Delta G$, GOLD domain deletion. e Sec14I3 interacts with VEGFR2 via its Sec14 domain. HEK293T cells were transfected with different Sec14I3 domain deletion forms with VEGFR2 plasmid respectively and harvested for IP. f Sec14I3 specifically interacts with RAB4A and RAB5A. Different RAB plasmids were co-transfected with VEGFR2 into HEK293T cells respectively for coIP assay. $\mathbf{g}$, h SEC14L2 interacts with RAB4 (g) and RAB5 (h) endogenously in HUVECs. i Sec14I3 interacts with RAB4A via its CRAL-TRIO and GOLD2 domains. Source data are provided as a Source Data file 
a

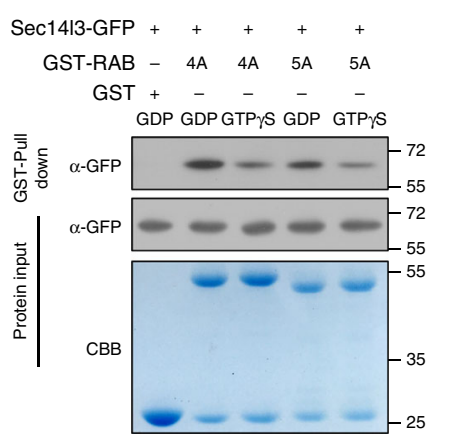

C

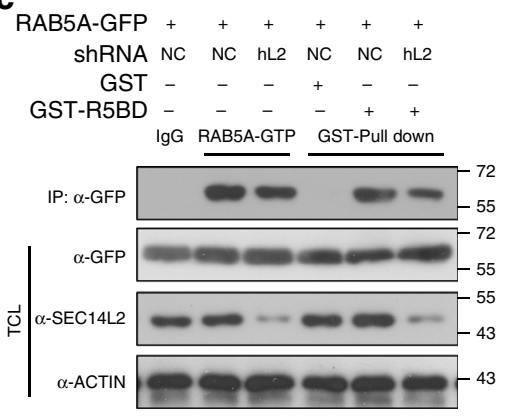

b
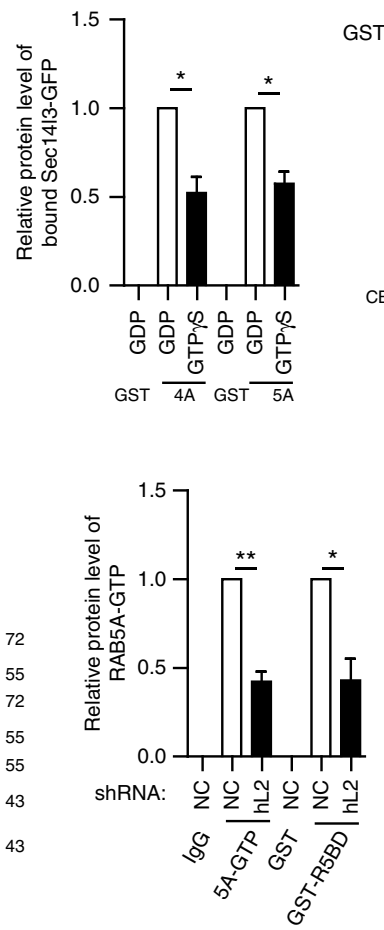

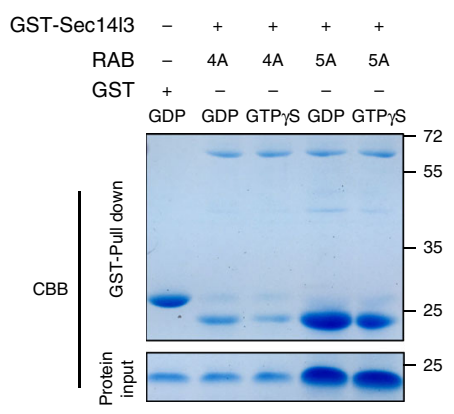

d
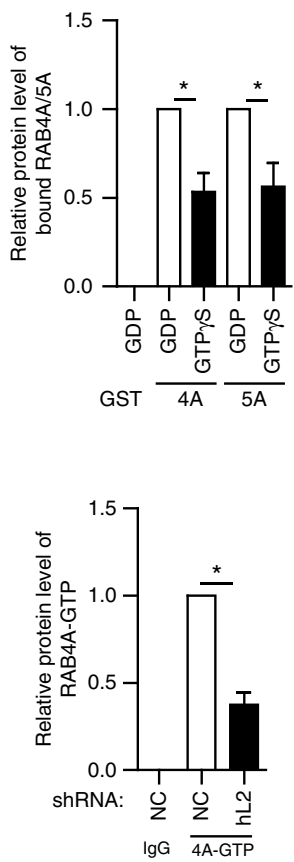

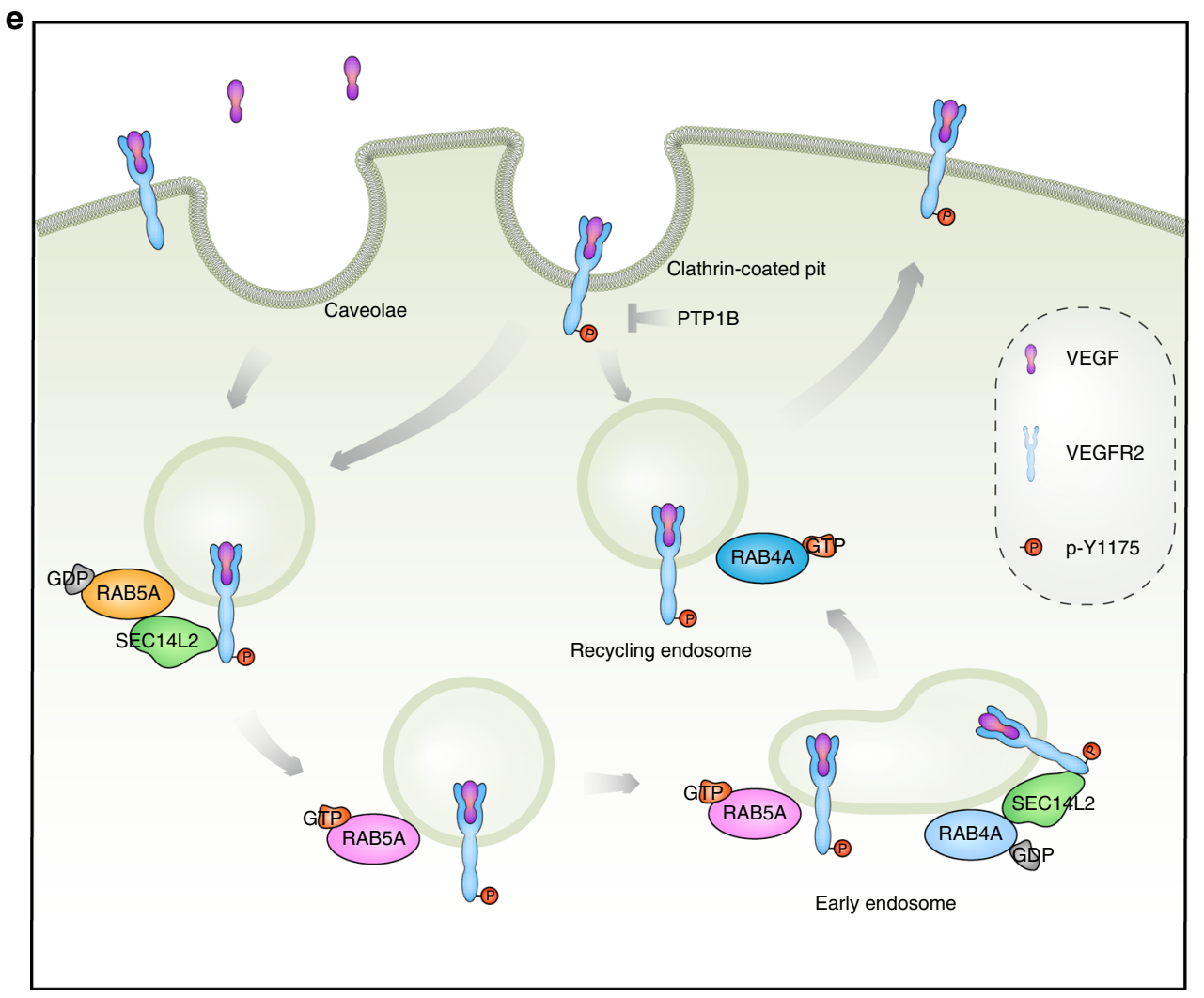

\section{Methods}

Zebrafish strains and embryos manipulation. Tuebingen strain of zebrafish (Danio rerio) was used. Unless otherwise stated, $T g(k d r l: G F P)^{s 843 T g}$ and $T g(f l i l a$ : $E G F P)^{y 1}$ transgenic lines were used for easy observation of vasculature development. Ethical approval is acquired from Tsinghua University Animal Care and Use Committee for the fish maintenance and manipulations, according to the institutional animal care and use committee (IACUC) protocol (AP\#13-MAM1) Embryos were raised and staged according to Kimmel et al. ${ }^{69}$. For injection, 1-cell stage embryos were harvested and injected with mRNAs or MOs in yolk for even distribution using the typical MPPI-2 quantitative injection equipment (Applied Scientific Instrumentation Co.). In this study, sec14l3, $c a-A K T$, and $c a-M E K$ mRNA were synthesized using the mMESSAGE mMACHINE Kit (Ambion AM1344) and purified using the RNeasy Mini Kit (Qiagen). Morpholinos were ordered from Gene Tools, LLC. and prepared in stocks. sec14l3-tMO2, sec14l3-sMO, ptp1btMO, ptplb-sMO and std-MO were synthesized by Gene Tools, LLC. The sequences of the used morpholinos are as follows: sec1413-tMO2: $5^{\prime}$-ATGTCGCCACGAGTGCAGCAGAAAT-3'; sec1413-sMO: 5'-ATGTTTCTCACCTCTCAGCCATCTG-3'; ptp1b-tMO: 5'-CGATTTCCCGAAACTCGGCTTCCAT-3'; 
Fig. 8 Sec14I3/SEC14L2 promote RAB5A/4A activation. a Sec14I3 interacts preferentially with GDP-bound state of RAB4A/5 A. HEK293T cells were transfected with Sec14/3-GFP and lysed for in vitro incubation with GTP $\gamma$ S/GDP loaded-RAB4A/5 A respectively. Quantification data of the relative protein levels of bound Sec14I3 are shown on the right $(n=3) .{ }^{*} p<0.05$. b Recombinant Sec14I3 protein purified from $E$. coli binds preferentially to GDP-bound $\mathrm{RAB} 4 \mathrm{~A} / 5 \mathrm{~A}$ in vitro. Recombinant GST-Sec14l3 protein purified from $E$. coli was loaded with GTP $\mathrm{S}$ and immobilized for incubation with the same amount of GTP $\gamma$ S/GDP loaded-RAB4A/5A. Quantification data of the relative protein levels of bound RAB4A/5A are shown on the right $(n=3)$. ${ }^{*} p<0.05$. c Knockdown of SEC14L2 alleviates RAB5A-GTP levels in HEK293T cells. NC or SEC14L2 shRNA transfected cell lysates were immunoprecipitated by an anti-RAB5A-GTP antibody or subjected to GST-R5BD pull down. Quantification of the relative protein level of RAB5A-GTP is shown on the right ( $n=3$ ). ${ }^{\star \star} p<0.01,{ }^{\star} p<0.05$. d Knockdown of SEC14L2 alleviates RAB4A-GTP level in HEK293T cells. NC- or SEC14L2 shRNA transfected HEK293T cell lysates were immunoprecipitated by anti-RAB4A-GTP antibody and quantification of the relative protein level of RAB4A-GTP is shown on the right $(n=3$ ). ${ }^{\star} p<0.05$. $t$-tests were used for statistical analyses in a-d. e Proposed working model of Sec14I3/SEC14L2 for RAB5A/4A-mediated VEGFR2 trafficking. Upon ligand stimulation, VEGFR2 could be internalized and recycled through RAB5A and RAB4A mediated pathways. Sec14I3/SEC14L2 are beneficial for increasing RAB5A/4A in their GTP-bound forms to accelerate the movement of VEGFR2-containing vesicles to early endosomes and subsequently recycling endosomes, consequently preventing VEGFR2 from PTP1B-mediated dephosphorylation

ptplb-sMO: 5'-GAAGCAGAATCAGTCTTTACCTGGT-3' and std-MO: 5'-CCT CTTACCTCAGTTACAATTTATA-3'. Every single embryo was injected with $1 \mathrm{nl}$ mRNA or MO solutions at indicated doses and raised to 19-30 hpf for observation. Embryos were harvested randomly for each experiment. For living imaging of vasculature development, chorion was removed from embryos by Pronase $\mathrm{K}$ (Sigma, $10 \mathrm{mg} \mathrm{ml}^{-1}$ ) and embryos were embedded in low melting agarose (Amresco 0815). Images were acquired using a Nikon A1RMPSi lasers scanning confocal microscope and processed by NIS-Element software for the measurement of diameters of vessels.

\section{Whole-mount in situ hybridization and immunofluorescence. Embryos at} indicated stages were harvested and fixed by $4 \%$ paraformaldehyde for at least $12 \mathrm{~h}$. After dehydration in methanol gradients, embryos could be stored at -20 degrees for future use. For WISH, at the 1st day, embryos were subjected for rehydration in $0.1 \%$ PBST buffer, pre-hybridization in $\mathrm{HYB}^{-}$buffer and hybridization with the indicated probes overnight at 65 degrees; At the 2 nd day, embryos were washed by $50 \%$ formamide/ 2x SSCT, 2x SSCT, 0.2x SSCT buffer sequentially. After blocking, embryos were incubated with Digoxigenin-AP antibody (11093274910, Roche, 1:3000) overnight at 4 degrees; At the 3rd day, after several rounds of washing by MABT buffer and staining buffer, embryos were transferred into 48-well plate and the substrate BM-Purple (11442074001, Roche) was added for probe visualization. Digoxigenin-UTP- ${ }^{-}$labeled sec14l3 antisense RNA probe was transcribed from a linearized plasmid in vitro. Stained whole embryos were photographed directly or sectioned in the transverse direction first (Leica CM1900) and then imaged by the Ds-Ril CCD camera under a Nikon SMZ1500 stereoscope. For immunofluorescence, mouse anti-GFP (sc-9996, Santa Cruz, 1:100), rabbit anti-p-Erk (Thr202/Tyr204) (\#9101, Cell Signaling Technology, 1:100), rabbit anti-p-Akt (Ser473) (\#4060, Cell Signaling Technology, 1:100) antibodies were used and embryos were imaged under Nikon AlRMPSi lasers scanning confocal microscope.

Cell culture and transfection. Plasmids used for cell transfection and their primers for construction are listed in Supplementary Table 1. HEK293T cells (Cell Resource Center, Peking Union Medical College) were cultured in DMEM (Life Technologies) supplemented with 10\% FBS (Hyclone, Logan, UT) and $50 \mathrm{mg} \mathrm{ml}^{-1}$ penicillin/streptomycin (PS) (Invitrogen). HUVECs (ScienCell \#8000) and HUAECs cells (ScienCell \#8010) were cultured in endothelial cell medium (ScienCell \#1001). Cell lines were checked for free of mycoplasma contamination by PCR and culture. Its species origin was confirmed with PCR. The identity of the cell line was authenticated with STR profiling (FBI, CODIS). The results for HEK293T cells can be viewed on the website (http://cellresource.cn). Transfections were performed using the polyethylenimine method for HEK293T cells. For endothelial cells, lentivirus infection method was used for knockdown experiments.

In vitro tube formation and wound closure assay. For in vitro tube formation assay, NC-shRNA or SEC14L2-shRNA infected HUVEC cells were plated on 12well plate $\left(2 \times 10^{5}\right.$ cells per well) covered by Matrigel (BD 356230) and cultured for assessment of tube formation following seeding. The tube number was counted. For wound closure assay, HUVEC cells were seeded in 6-well plate and grown to confluent state. Then, a 'scratch' was created using the P200 pipet tip and the closure of the wound was monitored after 24 or $48 \mathrm{~h}$ to reflect the migration of endothelial cells.

Internalization and recycling assay. To quantify internalization of VEGFR2, biotin based biochemical quantification and anti-VEGFR2 antibody-based immunofluorescence were adopted. The biotin based assay was performed essentially as described ${ }^{12}$. Generally, biotin in the culture medium can bind to proteins including VEGFR2 on the cell surface at $4^{\circ} \mathrm{C}$, but the biotin-labeled proteins would not be internalized at this temperature. After free biotin molecules are washed away and incubation temperature shifts to $37^{\circ} \mathrm{C}$, the biotin-labeled proteins on the cell surface start to move into the cytoplasm through internalization. The intracellular/ internalized biotin-labeled proteins can be pulled down using streptavidin beads after removal of those retained on the cell surface with iodoacetamide treatment and specific protein associated with biotin can be detected using a specific antibody at different time points of internalization (Supplementary Fig. 10). Briefly, transfected cells were washed twice using cold PBS $(\mathrm{pH} 8.0)$ and surface proteins were covalently labeled using a membrane-impermeant biotinylation reagent NHS-SSbiotin (Pierce, Rockford, IL) at $4{ }^{\circ} \mathrm{C}$ for $30 \mathrm{~min}$. After labeling, un-reacted biotin was washed away by chilled PBS and labeled cells were immediately incubated at $37^{\circ} \mathrm{C}$ to allow internalization for $5 \mathrm{~min}$ or $10 \mathrm{~min}$. After internalization, culture media was discarded and washed sequentially by $10 \%$ FBS twice, $1 \%$ BSA once and $20 \mathrm{mM}$ iodoacetamide (IAA) once for $10 \mathrm{~min}$ to remove biotin-labeled proteins remaining on the plasma membrane. Then, cells were lysed for streptavidin beads pull-down assay. For VEGFR2 immunofluorescence assay, HUVECs infected with shRNA were cultured for $72 \mathrm{~h}$ and then washed twice by cold PBS, which was followed by incubation with the Alexa647-conjugated anti-VEGFR2 antibody (\#359909 Biolegend, 1:200) for $30 \mathrm{~min}$ at $4^{\circ} \mathrm{C}$. After removing extra antibody, cells were placed in an incubator at $37^{\circ} \mathrm{C}$ for internalization. At indicated time points, cells were washed with acid PBS ( $\mathrm{pH} 2.5$ ) for three times to remove un-internalized anti-VEGFR2 antibody on the cell surface. After wash, cells were fixed by $4 \% \mathrm{PF}$ and subjected for immunofluorescence. Antibodies used here including SEC14L2 (TA503723, ORIGENE, 1:100), EEA1 (\#3288, Cell Signaling Technology, 1:100), VEGFR2 (\#2479, Cell Signaling Technology, 1:200) and CD63 (\#CBL553,

Millipore, 1:100).

To measure the recycling of VEGF2, transfected cells were washed and labeled at $4^{\circ} \mathrm{C}$ with biotin for $30 \mathrm{~min}$. The labeled cells were incubated with pre-warmed culture medium for $20 \mathrm{~min}$ at $22^{\circ} \mathrm{C}$ to allow cell surface proteins to internalize into RAB4-positive vesicles. The biotin-labeled proteins retained on the cell surface were removed by sequential washes with $10 \%$ FBS twice, $1 \%$ BSA once and $20 \mathrm{mM}$ iodoacetamide (IAA) once, each for $10 \mathrm{~min}$ at $4{ }^{\circ} \mathrm{C}$. Then cells were incubated at $37^{\circ} \mathrm{C}$ to allow recycling in the absence or presence of $100 \mathrm{ng} \mathrm{ml}^{-1}$ VEGFa for $15 \mathrm{~min}$. After recycling, cells were placed on ice and subjected to wash with $10 \%$ FBS twice, $1 \%$ BSA once and $20 \mathrm{mM}$ iodoacetamide (IAA) once, each for $10 \mathrm{~min}$, to remove labeled proteins that are returned to the plasma membrane. Finally, cells were lysed by TNE buffer for streptavidin beads pull-down assay and samples were boiled with $2 x$ loading buffer and prepared for western blotting.

Co-immunoprecipitation and GST pull-down. Co-IP was carried out according to the previous paper ${ }^{70}$. Briefly, cells were lysed using TNE buffer $(150 \mathrm{mM} \mathrm{NaCl}$ $5 \mathrm{mM}$ EDTA, $1 \%$ TritonX-100, $10 \mathrm{mM}$ Tris- $\mathrm{HCl}$ [pH 7.4]) mixed with protease inhibitors. The cell lysate was centrifuged at $120000 \times g$ to remove cell debris to acquire supernatant for subsequent SDS-PAGE or IP assay. For the co-IP assay, the following antibodies were used: anti-VEGFR2 (\#2479, Cell Signaling Technology, 1:200), anti-RAB4 (ab109009, Abcam, 1:100), anti-RAB5 (ab18211, Abcam, 1:100), anti-Flag (F1804, Sigma, 1:1000 for western blot). For immunoblotting analysis, anti-p-VEGFR2-Y1175 (\#2478, Cell Signaling Technology, 1:2000 for western blot), anti-p-VEGFR2-Y1054/1059 (ab5473, Abcam, 1:1000 for western blot), anti-GFP (sc-9996, Santa Cruz, 1:1000 for western blot), anti-SEC14L2 (TA503723, ORIGENE, 1:1000 for western blot) and anti-PTP1B (610139, BD Bioscience, 1:1000 for western blot). For GST pull-down assay, GST-Sec1413 and GST-RAB5A/4A recombinant proteins were expressed in $E$. coli. After $16 \mathrm{~h}$ induction by $1 \mathrm{mM}$ isopropyl $\beta$-D-1-Thiogalactopyranoside (IPTG, Takara 9030) at $16^{\circ} \mathrm{C}$, bacterial culture was centrifuged at $5000 \times g$ and washed by $50 \mathrm{mM}$ Tris- $\mathrm{HCl}(\mathrm{pH}$ 7.4) twice and then sonicated in $50 \mathrm{mM}$ Tris- $\mathrm{HCl}(\mathrm{pH}$ 7.4) solution supplemented with $1 \mathrm{mM}$ PMSF. Next, the supernatant was incubated with glutathione-Sepharose beads (GE Healthcare), washed by $50 \mathrm{mM}$ Tris- $\mathrm{HCl}(\mathrm{pH} 7.4), 50 \mathrm{mM}$ Tris- $\mathrm{HCl}(\mathrm{pH} 7.4)$ with $500 \mathrm{mM} \mathrm{NaCl}$ sequentially. After wash, about $1 \mathrm{ml}$ PreScission Protease (GE Healthcare) was added into GST-RAB5A/4 A beads to cut the GST tag and the resulting RAB5A/4 A protein was eluted using $50 \mathrm{mM}$ Tris- $\mathrm{HCl}(\mathrm{pH}$ 7.4) solution. For GTP $\gamma$ S/GDP- RAB5A/4 A pull-down assay, RAB5A/4 A protein was incubated in $20 \mathrm{mM}$ HEPES (pH 7.5), $100 \mathrm{mM} \mathrm{NaCl}, 10 \mathrm{mM}$ EDTA, $5 \mathrm{mM} \mathrm{MgCl}_{2}, 1 \mathrm{mM}$ DTT, and $1 \mathrm{mM}$ GTP $\gamma$ S/GDP (freshly made), for $90 \mathrm{~min}$ at room temperature to load GTP $\gamma$ S/GDP. Then GTP $\gamma$ S incubated GST-Sec14l3 beads were added into the solution for another $2 \mathrm{~h}$ incubation at $4^{\circ} \mathrm{C}$. Beads were finally washed and 
harvested for $2 \mathrm{x}$ loading buffer boiling and the samples were used for SDS-PAGE and Coomassie blue staining.

Statistical analysis. Results were analyzed with GraphPad Prism 5.0 and are presented as mean \pm SEM/SD as indicated, and comparisons were performed between two groups using a two-tailed Student's $t$-test or ANOVA test when comparing more than two conditions. For all analyses, ${ }^{*} p<0.05 ;{ }^{* *} p<0.01$; ${ }^{* * *} p<0.001$ were considered statistically significant; ns indicated statistical nonsignificance with $p>0.05$. Each experiment was carried out at least three times independently. The sample size is pre-estimated to ensure statistical analysis and no sample was optionally excluded from analysis. No blinding was done in the analyses and quantifications

\section{Data availability}

The authors declare that all data supporting the findings of this study are available within the article and its supplementary information files or from the corresponding author upon reasonable request. RNA-Seq raw data have been deposited in the GEO database under accession code: GSE126617 and other raw images are available at figshare.com for: Fig. 1d, https://doi.org/10.6084/m9.figshare.7729319, Fig. 2b, https://doi.org/10.6084/m9. figshare.7729325, Fig. 2g, https://doi.org/10.6084/m9.figshare.7729331, Fig. 3d, https:// doi.org/10.6084/m9.figshare.7729334, Fig. 3e, https://doi.org/10.6084/m9. figshare.7729337, Fig. 5e, https://doi.org/10.6084/m9.figshare.7729340. The source data underlying Figs. 2, 3, 4, 5, 6, 7 and Supplementary Fig. 4 are provided as a Source Data file.

Received: 27 February 2018 Accepted: 18 March 2019

Published online: 08 April 2019

\section{References}

1. Reichman-Fried, M. \& Raz, E. Blood, blebs and lumen expansion. Nat. Cell Biol. 18, 366-367 (2016).

2. Herbert, S. P. \& Stainier, D. Y. Molecular control of endothelial cell behaviour during blood vessel morphogenesis. Nat. Rev. Mol. Cell Biol. 12, 551-564 (2011).

3. Carmeliet, P. Angiogenesis in health and disease. Nat. Med. 9, 653-660 (2003).

4. Ramasamy, S. K., Kusumbe, A. P. \& Adams, R. H. Regulation of tissue morphogenesis by endothelial cell-derived signals. Trends Cell Biol. 25, 148-157 (2015).

5. Gore, A. V., Monzo, K., Cha, Y. R., Pan, W. \& Weinstein, B. M. Vascular development in the zebrafish. Cold Spring Harb. Perspect. Med. 2, a006684 (2012).

6. Ellertsdottir, E. et al. Vascular morphogenesis in the zebrafish embryo. Dev. Biol. 341, 56-65 (2010).

7. Lawson, N. D. \& Weinstein, B. M. In vivo imaging of embryonic vascular development using transgenic zebrafish. Dev. Biol. 248, 307-318 (2002).

8. Simons, M., Gordon, E. \& Claesson-Welsh, L. Mechanisms and regulation of endothelial VEGF receptor signalling. Nat. Rev. Mol. Cell Biol. 17, 611-625 (2016).

9. Roy, H., Bhardwaj, S. \& Yla-Herttuala, S. Biology of vascular endothelial growth factors. FEBS Lett. 580, 2879-2887 (2006).

10. Tammela, T., Enholm, B., Alitalo, K. \& Paavonen, K. The biology of vascular endothelial growth factors. Cardiovasc. Res. 65, 550-563 (2005)

11. Lohela, M., Bry, M., Tammela, T. \& Alitalo, K. VEGFs and receptors involved in angiogenesis versus lymphangiogenesis. Curr. Opin. Cell Biol. 21, 154-165 (2009).

12. Gampel, A. et al. VEGF regulates the mobilization of VEGFR2/KDR from an intracellular endothelial storage compartment. Blood 108, 2624-2631 (2006).

13. Hudson, N. et al. Differential apicobasal VEGF signaling at vascular bloodneural barriers. Dev. Cell 30, 541-552 (2014)

14. Saharinen, P. et al. Angiopoietins assemble distinct Tie2 signalling complexes in endothelial cell-cell and cell-matrix contacts. Nat. Cell Biol. 10, 527-537 (2008).

15. Fantin, A. et al. NRP1 acts cell autonomously in endothelium to promote tip cell function during sprouting angiogenesis. Blood 121, 2352-2362 (2013).

16. Koch, S. et al. NRP1 presented in trans to the endothelium arrests VEGFR2 endocytosis, preventing angiogenic signaling and tumor initiation. Dev. Cell 28, 633-646 (2014)

17. Gelfand, M. V. et al. Neuropilin-1 functions as a VEGFR2 co-receptor to guide developmental angiogenesis independent of ligand binding. Elife 3, e03720 (2014).

18. Di Fiore, P. P. \& De Camilli, P. Endocytosis and signaling. an inseparable partnership. Cell 106, 1-4 (2001).
19. Horowitz, A. \& Seerapu, H. R. Regulation of VEGF signaling by membrane traffic. Cell. Signal. 24, 1810-1820 (2012).

20. Sorkin, A. \& von Zastrow, M. Endocytosis and signalling: intertwining molecular networks. Nat. Rev. Mol. Cell Biol. 10, 609-622 (2009).

21. Nakamura, Y. et al. Role of protein tyrosine phosphatase $1 \mathrm{~B}$ in vascular endothelial growth factor signaling and cell-cell adhesions in endothelial cells. Circ. Res. 102, 1182-1191 (2008).

22. Lanahan, A. et al. The neuropilin 1 cytoplasmic domain is required for VEGFA-dependent arteriogenesis. Dev. Cell 25, 156-168 (2013).

23. Nakayama, M. et al. Spatial regulation of VEGF receptor endocytosis in angiogenesis. Nat. Cell Biol. 15, 249-260 (2013).

24. Wiedemann, C. \& Cockcroft, S. The Role of phosphatidylinositol transfer proteins (PITPs) in intracellular signalling. Trends Endocrinol. Metab. 9, 324-328 (1998).

25. Cockcroft, S. The diverse functions of phosphatidylinositol transfer proteins. Curr Top. Microbiol. Immunol. 362, 185-208 (2012).

26. Mousley, C. J., Davison, J. M. \& Bankaitis, V. A. Sec14 like PITPs couple lipid metabolism with phosphoinositide synthesis to regulate Golgi functionality. Subcell. Biochem. 59, 271-287 (2012).

27. Kempna, P. et al. Cloning of novel human SEC14p-like proteins: ligand binding and functional properties. Free Radic. Biol. Med. 34, 1458-1472 (2003).

28. Kauffmann-Zeh, A. et al. Requirement for phosphatidylinositol transfer protein in epidermal growth factor signaling. Science 268, 1188-1190 (1995)

29. Xie, Y. et al. Phosphatidylinositol transfer protein-alpha in netrin-1-induced PLC signalling and neurite outgrowth. Nat. Cell Biol. 7, 1124-1132 (2005).

30. Gong, B. et al. The Sec14-like phosphatidylinositol transfer proteins Sec14l3/ SEC14L2 act as GTPase proteins to mediate Wnt/Ca2+ signaling. Elife 6, e26362 (2017).

31. Sumanas, S. \& Lin, S. Ets1-related protein is a key regulator of vasculogenesis in zebrafish. PLoS Biol. 4, e10 (2006).

32. Manickam, V. et al. Regulation of vascular endothelial growth factor receptor 2 trafficking and angiogenesis by Golgi localized t-SNARE syntaxin 6. Blood 117, 1425-1435 (2011).

33. Robu, M. E. et al. p53 activation by knockdown technologies. PLoS Genet. 3, e78 (2007).

34. Herbert, S. P. et al. Arterial-venous segregation by selective cell sprouting: an alternative mode of blood vessel formation. Science 326, 294-298 (2009).

35. Kohli, V., Schumacher, J. A., Desai, S. P., Rehn, K. \& Sumanas, S. Arterial and venous progenitors of the major axial vessels originate at distinct locations. Dev. Cell 25, 196-206 (2013).

36. DeCicco-Skinner, K. L. et al. Endothelial cell tube formation assay for the in vitro study of angiogenesis. J. Vis. Exp. 91, e51312 (2014).

37. Tzima, E. et al. A mechanosensory complex that mediates the endothelial cell response to fluid shear stress. Nature 437, 426-431 (2005).

38. Hong, C. C., Peterson, Q. P., Hong, J. Y. \& Peterson, R. T. Artery/vein specification is governed by opposing phosphatidylinositol-3 kinase and MAP kinase/ERK signaling. Curr. Biol. 16, 1366-1372 (2006).

39. Robinson, M. J., Stippec, S. A., Goldsmith, E., White, M. A. \& Cobb, M. H. A constitutively active and nuclear form of the MAP kinase ERK2 is sufficient for neurite outgrowth and cell transformation. Curr. Biol. 8, 1141-1150 (1998).

40. Olsson, A. K., Dimberg, A., Kreuger, J. \& Claesson-Welsh, L. VEGF receptor signalling - in control of vascular function. Nat. Rev. Mol. Cell Biol. 7, 359-371 (2006).

41. Lanahan, A. A. et al. VEGF receptor 2 endocytic trafficking regulates arterial morphogenesis. Dev. Cell 18, 713-724 (2010).

42. Julien, S. G. et al. Protein tyrosine phosphatase 1B deficiency or inhibition delays ErbB2-induced mammary tumorigenesis and protects from lung metastasis. Nat. Genet. 39, 338-346 (2007).

43. Zhang, R. et al. Etk/Bmx transactivates vascular endothelial growth factor 2 and recruits phosphatidylinositol 3-kinase to mediate the tumor necrosis factor-induced angiogenic pathway. J. Biol. Chem. 278, 51267-51276 (2003)

44. Zerial, M. \& McBride, H. Rab proteins as membrane organizers. Nat. Rev. Mol. Cell Biol. 2, 107-117 (2001).

45. Galvez, T., Gilleron, J., Zerial, M. \& O’Sullivan, G. A. SnapShot: mammalian rab proteins in endocytic trafficking. Cell 151, 234-234 e232 (2012).

46. Scita, G. \& Di Fiore, P. P. The endocytic matrix. Nature 463, 464-473 (2010)

47. Stenmark, H. Rab GTPases as coordinators of vesicle traffic. Nat. Rev. Mol. Cell Biol. 10, 513-525 (2009).

48. Barr, F. \& Lambright, D. G. Rab GEFs and GAPs. Curr. Opin. Cell Biol. 22, 461-470 (2010).

49. Barr, F. A. Review series: rab GTPases and membrane identity: causal or inconsequential? J. Cell Biol. 202, 191-199 (2013).

50. Dou, Z. et al. Class IA PI3K p110beta subunit promotes autophagy through Rab5 small GTPase in response to growth factor limitation. Mol. Cell 50, 29-42 (2013).

51. Ballmer-Hofer, K., Andersson, A. E., Ratcliffe, L. E. \& Berger, P. Neuropilin-1 promotes VEGFR-2 trafficking through Rab11 vesicles thereby specifying signal output. Blood 118, 816-826 (2011). 
52. Gaengel, K. \& Betsholtz, C. Endocytosis regulates VEGF signalling during angiogenesis. Nat. Cell Biol. 15, 233-235 (2013).

53. Lampugnani, M. G., Orsenigo, F., Gagliani, M. C., Tacchetti, C. \& Dejana, E. Vascular endothelial cadherin controls VEGFR-2 internalization and signaling from intracellular compartments. J. Cell Biol. 174, 593-604 (2006).

54. Sawamiphak, S. et al. Ephrin-B2 regulates VEGFR2 function in developmental and tumour angiogenesis. Nature 465, 487-491 (2010).

55. Shin, M. et al. Vegfa signals through ERK to promote angiogenesis, but not artery differentiation. Development 143, 3796-3805 (2016).

56. Rossant, J. Vascular development and patterning: making the right choices. Curr. Opin. Genet. Dev. 13, 408-412 (2003).

57. Kim, S. H. et al. Vascular endothelial growth factor signaling regulates the segregation of artery and vein via ERK activity during vascular development. Biochem. Biophys. Res. Commun. 430, 1212-1216 (2013).

58. Deng, Y. et al. Endothelial RAF1/ERK activation regulates arterial morphogenesis. Blood 121, 3988-3996 S3981-3989 (2013).

59. Corson, L. B., Yamanaka, Y., Lai, K. M. \& Rossant, J. Spatial and temporal patterns of ERK signaling during mouse embryogenesis. Development 130, 4527-4537 (2003).

60. Zhang, C. et al. Inhibition of endothelial ERK signalling by Smad1/5 is essential for haematopoietic stem cell emergence. Nat. Commun. 5, 3431 (2014).

61. Bourdeau, A., Dube, N. \& Tremblay, M. L. Cytoplasmic protein tyrosine phosphatases, regulation and function: the roles of PTP1B and TC-PTP. Curr. Opin. Cell. Biol. 17, 203-209 (2005).

62. Mellberg, S. et al. Transcriptional profiling reveals a critical role for tyrosine phosphatase VE-PTP in regulation of VEGFR2 activity and endothelial cell morphogenesis. FASEB J. 23, 1490-1502 (2009).

63. Grazia Lampugnani, M. et al. Contact inhibition of VEGF-induced proliferation requires vascular endothelial cadherin, beta-catenin, and the phosphatase DEP-1/CD148. J. Cell Biol. 161, 793-804 (2003).

64. Pelster, B. \& Burggren, W. W. Disruption of hemoglobin oxygen transport does not impact oxygen-dependent physiological processes in developing embryos of zebra fish (Danio rerio). Circ. Res. 79, 358-362 (1996).

65. McMahon, H. T. \& Boucrot, E. Molecular mechanism and physiological functions of clathrin-mediated endocytosis. Nat. Rev. Mol. Cell Biol. 12, 517-533 (2011).

66. De Matteis, M. A. \& Godi, A. PI-loting membrane traffic. Nat. Cell Biol. 6, 487-492 (2004).

67. Rink, J., Ghigo, E., Kalaidzidis, Y. \& Zerial, M. Rab conversion as a mechanism of progression from early to late endosomes. Cell 122, 735-749 (2005).

68. Jean, S., Cox, S., Schmidt, E. J., Robinson, F. L. \& Kiger, A. Sbf/MTMR13 coordinates PI(3)P and Rab21 regulation in endocytic control of cellular remodeling. Mol. Biol. Cell 23, 2723-2740 (2012).

69. Kimmel, C. B., Ballard, W. W., Kimmel, S. R., Ullmann, B. \& Schilling, T. F. Stages of embryonic development of the zebrafish. Dev. Dyn. 203, 253-310 (1995).

70. Zhang, Y. et al. Rock2 controls TGFbeta signaling and inhibits mesoderm induction in zebrafish embryos. J. Cell Sci. 122, 2197-2207 (2009).

\section{Acknowledgements}

We thank Drs. Nathan D. Lawson (University of Massachusetts Medical School) and Bo Zhang (Peking University) for providing transgenic fishes and Wang Min (Yale University) for providing WT-VEGFR2 and KM-VEGFR2 plasmids. We also appreciate the helpful discussion and technical assistance from members of the Meng Laboratory. This work was financially supported by grants from the National Natural Science Foundation of China (\#31522035, \#91754112, \#31801130 and \#31371460), Major Science Programs of China (\#2012CB945100).

\section{Author contributions}

B.G. performed most experiments, collected and organized data. Z.H.L. helped to culture cells for partial biochemical analysis. W.H.X., G.Y.L., and S.H.D. participated in a part of the work, including whole-mount in situ hybridization, constructs cloning and so on. A.M.M. and S.J.J. proposed and managed the project, and B.G., A.M.M., and S.J.J. designed the study and prepared the manuscript.

\section{Additional information}

Supplementary Information accompanies this paper at https://doi.org/10.1038/s41467019-09604-0.

Competing interests: The authors declare no competing interests.

Reprints and permission information is available online at http://npg.nature.com/ reprintsandpermissions/

Journal peer review information: Nature Communications thanks Shamshad Cockcroft and the other anonymous reviewer(s) for their contribution to the peer review of this work. Peer reviewer reports are available.

Publisher's note: Springer Nature remains neutral with regard to jurisdictional claims in published maps and institutional affiliations.

(c) (i) Open Access This article is licensed under a Creative Commons Attribution 4.0 International License, which permits use, sharing, adaptation, distribution and reproduction in any medium or format, as long as you give appropriate credit to the original author(s) and the source, provide a link to the Creative Commons license, and indicate if changes were made. The images or other third party material in this article are included in the article's Creative Commons license, unless indicated otherwise in a credit line to the material. If material is not included in the article's Creative Commons license and your intended use is not permitted by statutory regulation or exceeds the permitted use, you will need to obtain permission directly from the copyright holder. To view a copy of this license, visit http://creativecommons.org/ licenses/by/4.0/.

(C) The Author(s) 2019 